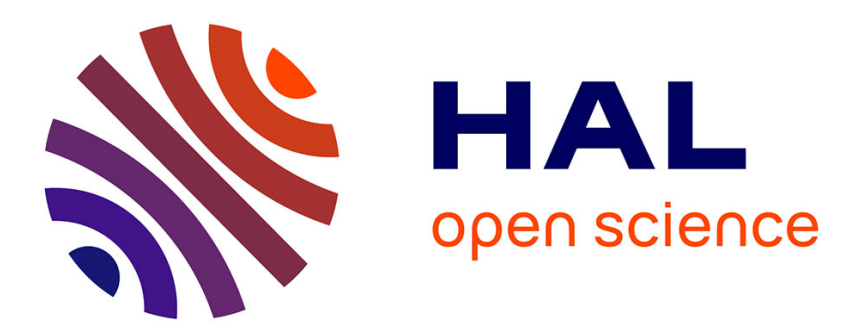

\title{
La viticulture antique en Languedoc-Roussillon
}

Loïc Buffat, Christophe Pellecuer, Stéphane Mauné, Hervé Pomarèdes

\section{To cite this version:}

Loïc Buffat, Christophe Pellecuer, Stéphane Mauné, Hervé Pomarèdes. La viticulture antique en Languedoc-Roussillon. Gallia - Archéologie de la France antique, 2001, La viticulture en Gaule, 58, pp.91-111. 10.3406/galia.2001.3175 . hal-01910050

\section{HAL Id: hal-01910050 \\ https://hal.science/hal-01910050}

Submitted on 20 Jan 2020

HAL is a multi-disciplinary open access archive for the deposit and dissemination of scientific research documents, whether they are published or not. The documents may come from teaching and research institutions in France or abroad, or from public or private research centers.
L'archive ouverte pluridisciplinaire HAL, est destinée au dépôt et à la diffusion de documents scientifiques de niveau recherche, publiés ou non, émanant des établissements d'enseignement et de recherche français ou étrangers, des laboratoires publics ou privés.

\section{(1) (1) $\$$}

Distributed under a Creative Commons Attribution - NonCommercial - NoDerivatives| 4.0 


\title{
LA VITICULTURE ANTIQUE EN LANGUEDOC-ROUSSILLON
}

\author{
Loïc Buffat, Christophe Pellecuer \\ avec des contributions de Stéphane Mauné et Hervé Pomarèdes
}

\begin{abstract}
Mots-clés. Nîmes, Béziers, Ruscino, Narbonnaise, amphores gauloises, dolium, villa.
Key-words. Nîmes, Béziers, Ruscino, Gallia Narbonensis, Gallic amphorae, dolium, villa.

Résumé. La viticulture d'époque gallo-romaine, pour la partie de la Narbonnaise correspondant à l'actuelle région du LanguedocRoussillon, ne pouvait être évoquée jusqu'à une date récente qu'au moyen des seules études menées sur les ateliers de fabrication d'amphores. Les installations de production vinicole sont maintenant mieux connues grâce aux fouilles de plus en plus fréquentes d'établissements ruraux. Une douzaine de ces sites, villae el fermes plus modestes mises au jour dans les départements du Gard, de l'Hérault et des Pyrénées-Orientales, est présentée sous la forme de notices. Cies nouvelles données permeltent de préciser les grandes lignes de l'évolution de la viticulture provinciale, depuis la période de la conquête jusqu'au début du haut Moyen Âge. Les premiers signes d'une intensification de la production dans le territoire des cités de Nîmes et de Béziers sont nettement perceptibles autour du changement d'ère, prélude à un développement sans prérédent durant tout le Haut-Empire. Des témoignages matériels de plus en plus nombreux confirment sans surprise la poursuite de la production après le III' s., mais le poids réel de la viticullure dans l'économie agricole régionale, pour la période de l'Antiquité tardive, demande encore à être précisé.
\end{abstract}

\begin{abstract}
Until recenlly, Gallo-Roman viticullure in the modern region of Languedoc-Roussillon situated in Narbonensis, has only been studied through investigations carried out on amphorce workshops. Now the wine production siles are better known thanks to an increasing number of excavations going on rural settlements. A dozen of these sites, villae and smaller farms recovered in the Gard, Hérault and Pyrénées-Orientales departments is depicted below. These new discoveries allow to give an outline of the historical development of provincial viticulture from the Conquest up to the beginning of the Early Middle Ages. The first signs showing the increase of the production in the territories of Nîmes and Béziers are strongly discernible at the turn of the $1^{\text {rt }}$ century, prelude to a major development during the Early Empire. Numerous facts obviously confirm that production goes on after the $3^{\text {rd }}$ century, but the impact of the viticulture in the regional agricultural economy during the Late Antiquity needs to be more precisely examined.
\end{abstract}

Les vins de la Narbonnaise n'étaient pas de grands crus, si l'on se fie aux dires de Pline qui les accusaient d'être pour beaucoup frelatés selon divers procédés (Pline, H. N., XIV, 68). Seul le vin de Béziers échappait quelque peu à ce jugement général, mais sa qualité le plaçait bien en deçà du vin marseillais, référence obligée entre Alpes et Pyrénées. La réputation du vin biterrois restait circonscrite, selon l'auteur antique, à l'échelle des Gaules (id., ibid.). Pourtant, même s'il ne comptait pas parmi les meilleurs, ce vin est diffusé à Rome dès la première moitié $d u I^{\text {er }} s$. de notre ère, comme le montrent deux amphores Dressel 2/4 découvertes dans un dépôt du Castro Pretorio. Les inscriptions qu'elles portent nous indiquent clairement l'origine mais aussi la nature des contenus: un vin de garde, âgé de cinq ans, et un blanc (CIL XV, 4542 et 4543). D'autres tituli picti permettent de savoir que l'on élevait dans cette cité de la Narbonnaise occidentale un cépage aussi 
réputé que l'Aminée (Liou, Marichal, 1978, $\mathrm{n}^{\circ} 81$, p. 179-181). Tout porte à croirc qu'un certain succès du vin biterrois n'est pas étranger à la large diffusion dans l'empire des amphores gauloises à fond plat du type Gauloise 4, à partir de la seconde moitié du I ${ }^{\mathrm{er}}$ s. de notre ère (sur l'ensemble de ces questions, voir Laubenheimer, 1985).

Les origines antiques du vin biterrois ainsi que le passé viticole récent de la région languedocienne pourraient laisser penser que l'on s'est attaché très tôt à retrouver et à étudier les traces des installations de production de la partic occidentale de la Narbonnaise. Un rapide tour d'horizon bibliographique montre que la monoculture de la vigne, entre le milieu du XIX's. et la dernière guerre mondiale, n'a pas été en fait un contexte favorable à l'émergence d'une véritable recherche de terrain. Ce sont les mêmes contraintes et carences qui ont pesé pendant longtemps sur l'archéologie de la villa (Pellecuer dir., 1996). Il faut attendre les années soixante-dix pour voir prendre corps une réflexion d'ensemble sur les amphores vinaires sud-gauloises dans laquelle le Languedoc-Roussillon tient une place de premier ordre (Laubenheimer, 1977). Vingt ans plus tard, on recense à l'ouest du Rhône vingt-sept ateliers liés à la fabrication de ces conteneurs sur la cinquantaine connuc cn Narbonnaisc (Laubenheimer, 1998a, p. 166-167). L'officine du Clos de Raynaud à Sallèlesd'Aude (Aude) est à ce jour la fabrique qui a été explorée et étudiée de la façon la plus exhaustive (voir en dernier lieu Laubenheimer dir., 2001). Les résultats accumulés dans le domaine du conditionnement soulignaient d'autant plus les lacunes criantes pour tout ce qui touche à la production et à la vinification. Jusqu'à il y a peu, on devait se contenter d'une vision très partielle - le plus souvent des cuves ou des blocs de pressoir isolés ou quelques dolia. La ferme de La Combe de Fignols à Péret (Hérault), fouillée entre 1975 et 1983 (Olive, 1989), est restée pendant un temps le seul exemple à notre disposition d'une installation de production complète, même si la destination exacte des équipements dégagés - vin ou huile ? - prête encore à discussion (Brun, 1993a, p. 326, note 55).

Ce déséquilibre dans la documentation tend aujourd'hui à être compensé grâce au regain des études sur l'espace rural gallo-romain, du fait d'un démarrage somme toute assez récent d'une archéologie préventive au caractère systématique. Lors de la table ronde

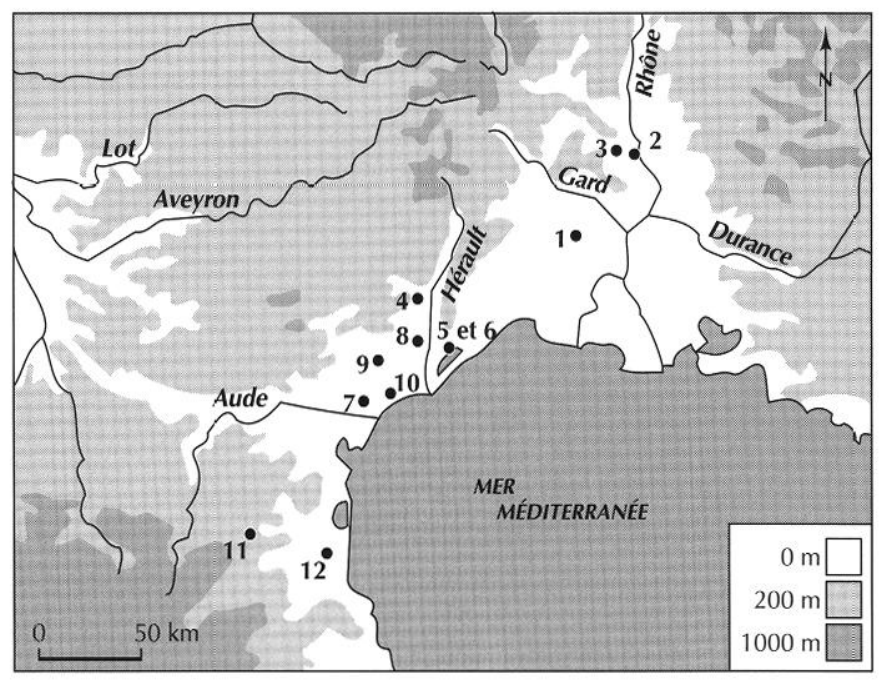

Fig. 40 - Carle des principales installations de production viticole fouillées en Languedoc-Roussillon.

GARl) : 1, Sainl-André-de-Codols, Nîmes ; 2, La Ramière, Roquemaure ; 3, Mayran, Saint-Victor-la-Coste.

HÉRAULT: 4, La Quinlarié, Clermont-l'Hérault ; 5, Les Prés-Bas, Loupian ; 6, Le Bourbou/Port-de-Loupian, Loupian;

7, Les Farguettes, Nissan-lez-linsérune; 8, Vareilles, Paulhan/Adissan; 9, Les Jurières-Basses, Puissalicon;

10, La Domergue, Sauvian.

PYRÉNÉES-ORIENTALES : 11, Pla de l'Aïgo, Caramany; 12, Le Petit Clos, Perpignan.

organisée à Lattes en 1998 (Buffat, Pellecuer, 1998) ${ }^{130}$, on a pu prendre la mesure des progrès accomplis, d'abord d'un point de vue documentaire, mais aussi sur un certain nombre de thèmes. Il est aujourd'hui possible de souligner avec plus de force le caractère précoce de la viticulture locale, plus précoce même que ne le laissait supposer la chronologie des amphores sud-gauloises, de décrire plus précisément le développement du HautEmpire et de distinguer les premiers indices en faveur d'unc pcrmancnce de la production durant l'Antiquité tardive. Ces réflexions ont servi de point de départ à la présente contribution.

130. Ont participé à cettc table ronde à l'initiative de l'UMR 154 du CNRS et du ministère de la Culture, C. Arlaud, A. Avril, F. Bazile, J.-P. Brun, L. Buffat, A. Chartrain, D. Goury, M. Fcugère, V. Forest, P. Garmy, O. Ginouve', J. Kotarba, M. Jamet, F. Laubenheimer, E. Llopis, M. Lugand, S. Mauné, C. Pellecuer, H. Pomarèdes, K. Roger et L. Vidal. 

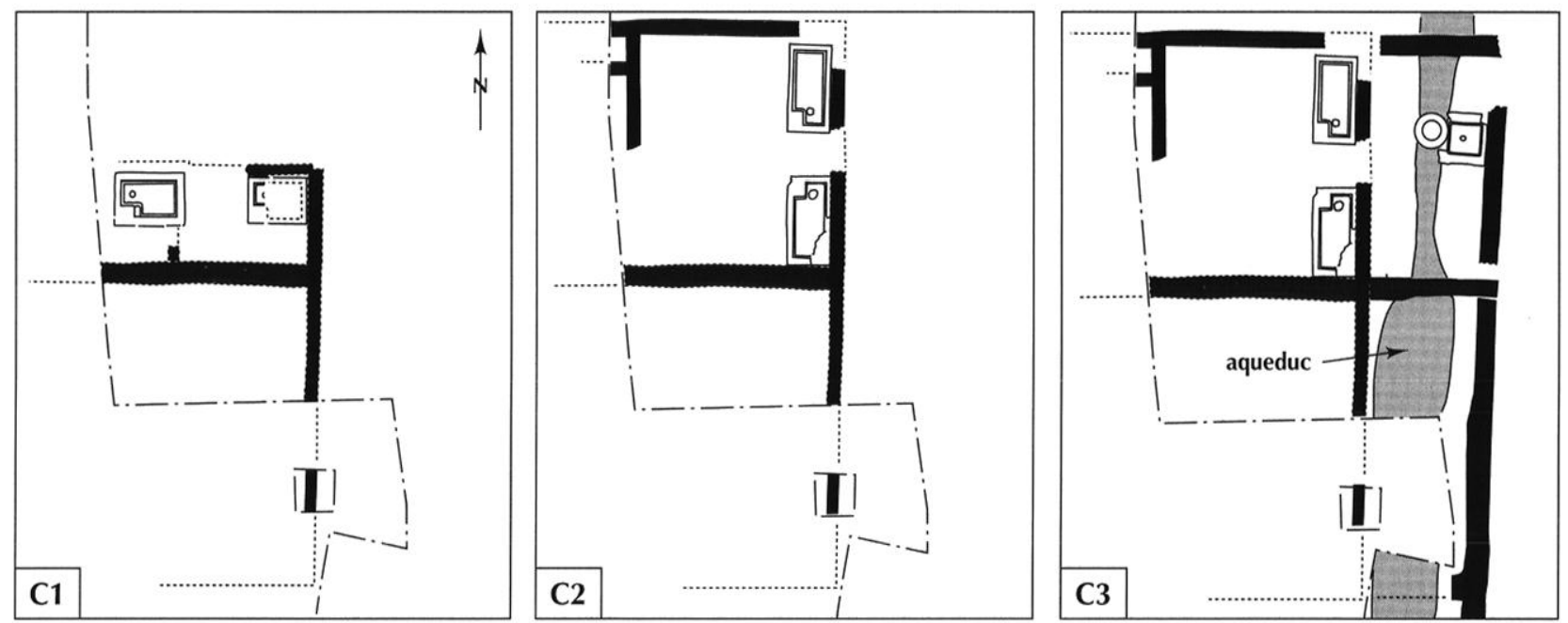

$\underset{0}{0}$ $5 \mathrm{~m}$

Fig. 41 - Les installations vinicoles de la villa de Mayran à Saint-Victor-la-Coste, Gard: C1, état du III s. ; C2, état du IV s. ; C3, état final, utilisé jusqu'à la fin du V's. ou le début du VTe s. (dessin L. Buffat).

\section{LES PRINCIPALES INSTALLATIONS DE PRODUCTION FOUILLÉES EN LANGUEDOC-ROUSSILLON (fig. 40)}

\section{DÉPARTEMENT DU GARD}

\section{MAYran À SAINT-Victor-LA-Coste}

Un complexe vinicole de l'Antiquité tardive (fig. 41) a été découvert sur la grande villa de Mayran à l'occasion d'une fouille de sauvetage réalisée entre 1995 et 1997 par L. Buffat et H. Petitot (Buffat, 1996). Des cuves ont été dégagées dans l'une des pièces du bâtiment. Les deux plus anciennes ont apparemment été mises en place au III $^{e}$ s. (C1). En témoigneraient deux fragments d'amphores africaines retrouvées dans les radiers. Elles s'organisent selon un axe est-ouest. Elles font place durant le $\mathrm{IV}^{\mathrm{e}} \mathrm{s}$. à deux autres cuves d'axe nord-sud (C2). Les revêtements de parois ont été repris à une date indéterminée (C.3). Ces cuves ont visiblement fonctionné jusqu'à la fin $\mathrm{du} \mathrm{V}^{\mathrm{e}}$ ou au début du $\mathrm{VI}^{\mathrm{e}} \mathrm{s}$. Elles sont toutes équipées de marches d'escalier. Il est difficile d'évaluer leur capacité étant donné l'arasement des vestiges. On estime néanmoins que les plus petites avaient une capacité de l'ordre de 30 à $35 \mathrm{hl}$, les plus grandes de 50 à $60 \mathrm{hl}$. Ces estimations indiquent $a$ priori une plus forte capacité des cuves les plus anciennes. Le site, extrêmement arasé, n'a conservé aucune trace de fouloir ou de pressoir. Un vignoble, à proximité immédiate de la villa, serait attesté par une quinzaine de petites fosses de plantation, de forme allongée et remplies de galets.

\section{LA RAMiÈre À Roquemaure}

La villa de La Ramière a été fouillée en 1996 sous la direction de $\mathrm{H}$. Pomarèdes, $\mathrm{H}$. Petitot et $\mathrm{O}$. Maufras, dans le cadre des travaux archéologiques menés sur le tracé du TGV Méditerranée (Barberan et al., à paraître).

Les premiers bâtiments remontent à l'époque augustéenne, mais aucun élément ne permet d'envisager la production de vin dès cette époque. Dans le deuxième quart du $\mathrm{I}^{\text {er }} \mathrm{s}$. après $\mathrm{J}$.-C., les constructions les plus anciennes sont détruites pour faire place à une ferme. On trouve, dans la partie est du nouvel ensemble, une série de pièces, aux maçonneries liées au mortier, qui se rapporterait à une activité vinicole. Deux d'entre elles comportent un sol de tuileau et pourraient être des fouloirs ou des cuves de recueil. Une autre pièce livre un four de plan bielliptique, aux parois de briques, qui serait destiné à la préparation du defrutum ${ }^{131}$. Ces hypothèses

131. Selon l'interprétation proposée par Jean-Pierre Brun. 


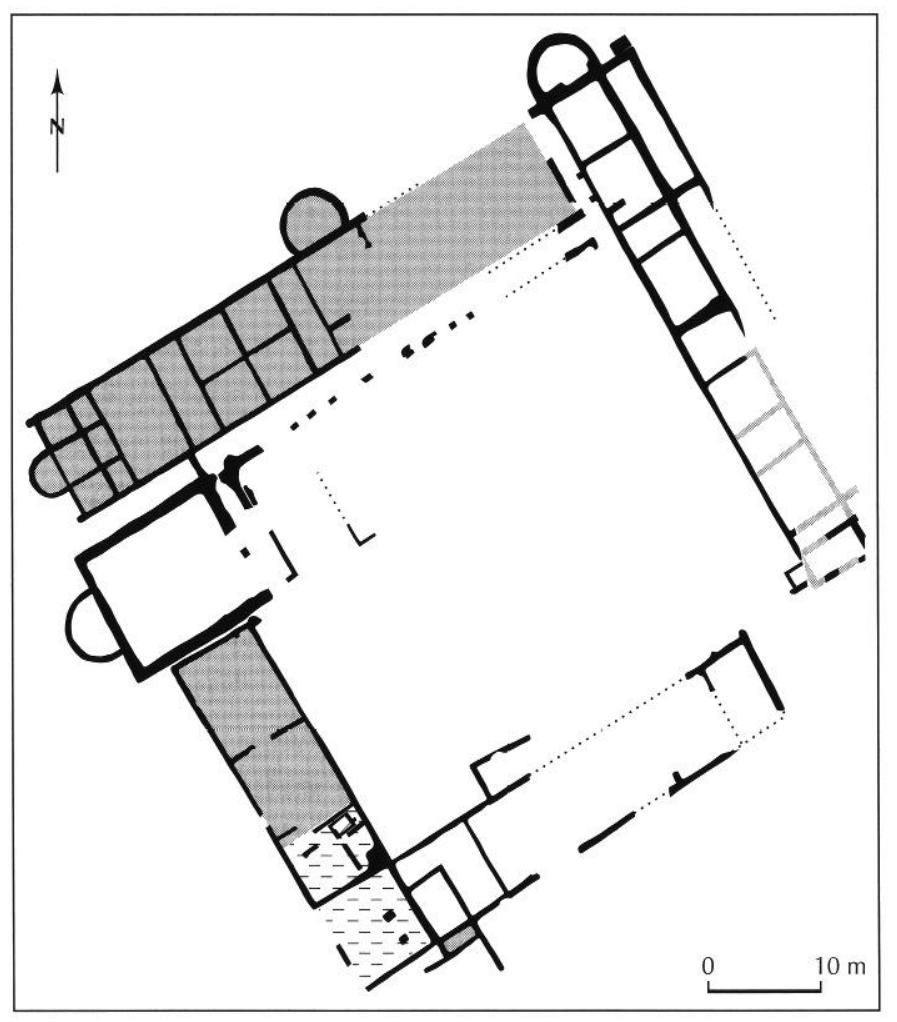

Fig. 42 - La villa de Saint-André-de-Codols à Nîmes (Gard), élat 3, milieu III s.-IV s. : en grisé, les espaces utilisables pour le stockage; en hachuré, les installations de transformation (dessin P. Garczynski, CEPAM-CNRS d'après S. Barberan, complété).

restent cependant à confirmer par une étude plus détaillée des structures dégagées.

Durant la seconde moitié du $\mathrm{I}^{\mathrm{er}} \mathrm{s}$., l'établissement se développe considérablement et atteint alors une surface de $5000 \mathrm{~m}^{2}$ environ. La villa s'organise selon plusieurs cours. Au sud, se trouve la pars fructuaria, caractérisée par un cellier installé selon un axe est-ouest. Celui-ci comporte 24 récipients (Barberan et al., à paraître). En retenant une capacité de $15 \mathrm{hl}$ par récipient, le chai aurait une contenance de $360 \mathrm{hl}$.

Le second cellier d'axe nord-sud est probablement construit entre la fin du $\mathrm{I}^{\mathrm{er}} \mathrm{s}$. et le début du $\mathrm{II}^{\mathrm{e}} \mathrm{s}$. La cella vinaria initiale est en partie détruite par ce nouveau bâtiment, mais sa partic orientale reste apparemment en fonctionnement. Le dispositif ainsi créé présente un plan en « $\mathrm{L}$ » et une capacité de 44 à 52 dolia, représentant un volume global de 660 à $780 \mathrm{hl}$. Il n'y a sur le site aucune trace de pressoir (contrepoids ou blocs d'ancrage), certainement à cause de l'arasement des vestiges. À une quinzaine de mètres au nord-est des celliers, existe néanmoins une cuve à cupule et cordons d'étanchéité, qui s'apparente au type courant des cuves de recueil. Il a été également dégagé un vaste sol de tuileau (environ $80 \mathrm{~m}^{2}$ ) présentant vers l'est une importante dépression. Ce dispositif assez inhabituel pourrait appartenir à un fouloir.

Entre la fin $d u \mathrm{II}^{e}$ s. et le milieu du $\mathrm{III}^{e}$ s., la villa connaît une importante campagne d'agrandissement. Le chai de la période antérieure n'est plus utilisé après le $\mathrm{III}^{e}$ s. Il existe sur le site encore au $\mathrm{IV}^{e} \mathrm{~s}$. de grands bâtiments de stockage, mais aucun élément ne permet pour le moment de supposer une continuité de la production viticole durant l'Antiquité tardive.

\section{SAINT-ANDRÉ-DE-COdOLS À NîMES}

La villa de Saint-André-de-Codols (fig. 42) a fait l'objet d'une fouille extensive en 1995, sous la direction de H. Pomarèdes, avec la collaboration de O. Maufras et L. Sauvage (Pomarèdes, in: Pellecuer dir., 1996). Aucun chai n'a été individualisé pour les premiers bâtiments mis en place au II $\mathrm{e}$. À la fin du $\mathrm{III}^{\mathrm{e}} \mathrm{s}$. ou au début du IV $\mathrm{s}$., l'établissement rural est radicalement transformé. Quatre corps de bâtiment sont disposés autour d'une vaste cour centrale et forment un quadrilatère de $90 \mathrm{~m}$ de côté. L'aile occidentale a livré les restes d'une cuve dont la capacité est estimée à $60 \mathrm{hl}$. Elle est bordée au sud d'un radier formé de plusieurs niveaux de blocs, interprété comme les fondations d'un pressoir ou, en l'absence de traces explicites de machinerie, comme un fouloir. Dans une pièce voisine, deux bases maçonnées pourraient marquer l'emplacement d'un autre pressoir. Néanmoins, l'espacement important entre les deux massifs de maçonnerie invite plutôt à en restituer deux. Aucun cellier à dolia n'a été repéré sur le site. Des surfaces de stockage sont à chercher dans les pièces contiguës aux pressoirs mais aussi au rez-de-chaussée de l'aile nord-est, sous la forme de magasins qui devaient supporter un étage résidentiel.

\section{DÉPARTEMENT DE L'HÉRAULT}

\section{LA DOMERgue à SAUVIAN}

Connue depuis le début du siècle, la grande villa de La Domergue a fait l'objet en 1994 d'une importante campagne de fouille préventive (Ginouvez, 1995). 


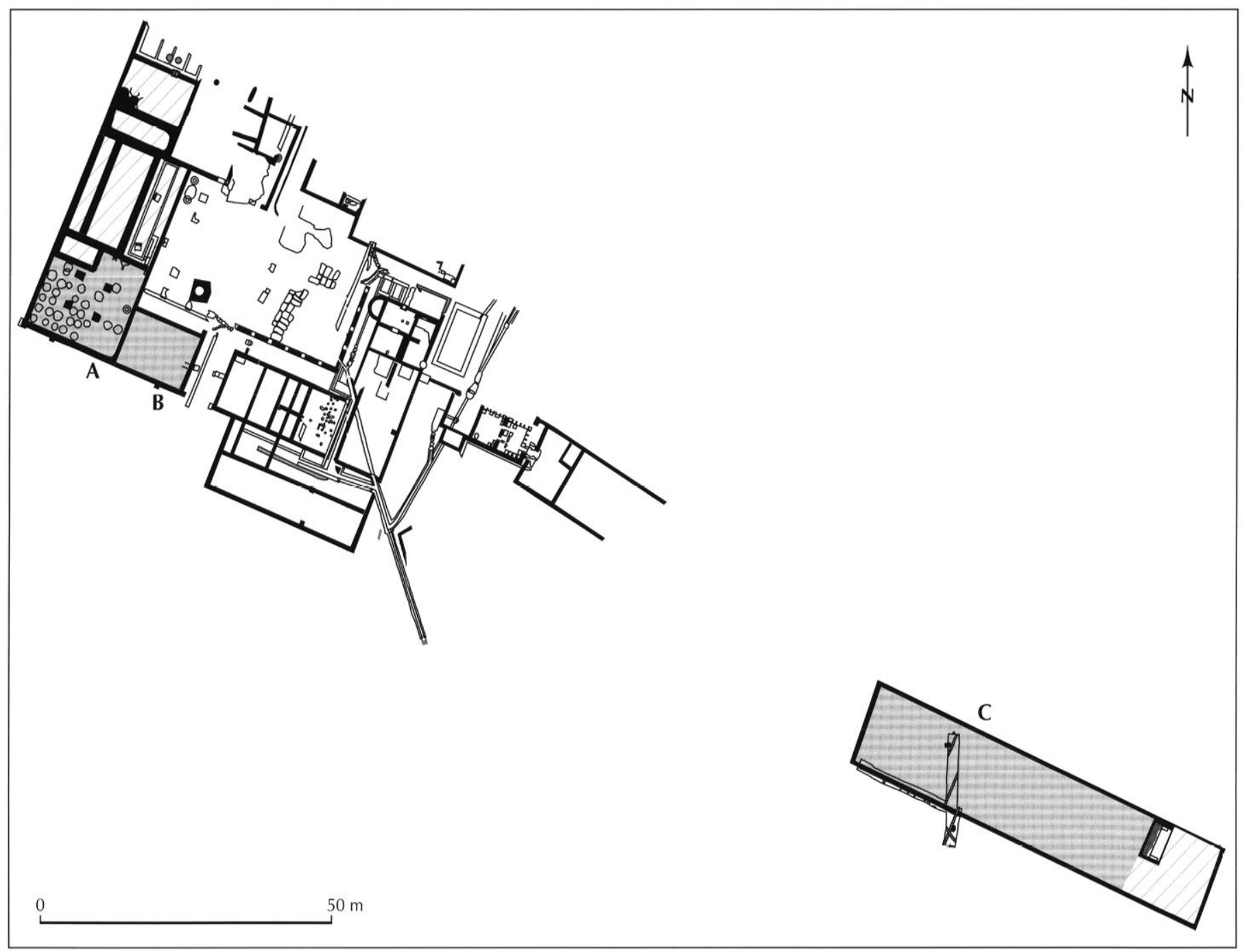

Fig. 43 - Une grande villa de la Narbonnaise occidentale, La Domergue à Sauvian (Hérault) :

en grisé, les différents espaces de stockage (A à C); en hachuré, les pressoirs (dessin L. Vidal d'après $O$. Ginouvez, complété).

L'établissement rural, exploré préalablement à la réalisation d'une ZAC, a été décapé sur une surface de $3500 \mathrm{~m}^{2}$ (fig. 43). Autour du noyau bâti, des tranchées ont permis de révćler des ćlćments du parccllairc antique, dcux chemins - dont un antérieur à la construction de la villa -, ainsi que des traces de défoncement en relation avec la culture de la vigne (Vidal, 1998). Une chronologie précise des différentes phases d'occupation du site n'a pas pu être établie dans le cadre de la fouille d'urgence. L'essentiel de cette occupation est à situer entre le $\mathrm{I}^{\text {er }}$ et le $\mathrm{V}^{\mathrm{e}} \mathrm{s}$. et des traces d'une fréquentation durant la période médiévale ont été reconnues.

La villa est organisée autour d'une vaste cour centrale. Dans l'aile orientale prend place l'espace résidentiel dont on n'a reconnu que le complexe thermal. La partie ouest de la villa est clairement dévolue aux activités de transformation agricoles et plus particulièrement à la vinification. Unc grandc sallc dc prcssuragc, mcsurant $14,6 \mathrm{~m}$ x 5,8 $\mathrm{m}$, a été dégagée. Des aménagements intérieurs ne subsiste qu'un épais radier de blocs d'une puissance de 1,2 $\mathrm{m}$. La présence de deux contrepoids, en place dans la salle de manœuvre située à l'est, invite à restituer au moins deux pressoirs. Les jus de presse s'écoulaient vers le sud en direction d'une grande cuve. Celle-ci est installée dans un premier cellier de plan carré qui abrite 62 dolia, dont plusieurs encore en place (fig. 43, A). Malgré une fouille très partielle, une grande salle mitoyenne, dotée de contreforts, doit être également 
interprétée comme un cellier (fig. 43, B). Des fosses circulaires sont signalées à l'intérieur du bâtiment. La capacité de ce cellier est fixée entre 16 et 32 dolia, le chiffre le plus probable étant de 27 récipients (Vidal, 1998, p. 43). Au nord du pressoir, quatre cuves dont les fonds sont en dénivelé graduel ont été dégagées. Cette disposition évoque un système de décantation par gravité, qui caractérise habituellement les installations oléicoles.

Un troisième cellier a été repéré une cinquantaine de mètres au sud-est de la villa (fig. 43 , C). Mesurant $12,70 \mathrm{~m}$ sur $59 \mathrm{~m}$, il constitue la plus grande unité de stockage reconnue sur le site. L'intérieur, décapé sur une petite surface, n'a livré qu'une grande cuve. Le bâtiment pouvait accucillir entre 99 et 189 vaisseaux, mais un chiffre moyen de l'ordre de 163 dolia peut être retenu en tenant compte de la densité des récipients observés dans le premier cellier (Vidal, 1998, p. 43).

Les capacités théoriques des différents chais permettent d'estimer la surface occupée par le vignoble dans ce domaine dont l'étendue est fixée à 500 ha. En retenant l'hypothèse du stockage d'une scule vendange, les celliers auraient contenu au maximum le produit de 63 à 73 ha de vignes (rendement de $30 \mathrm{hl} / \mathrm{ha}$ ), au minimum celui de 31 à 36 ha (rendement de $60 \mathrm{hl} / \mathrm{ha}$ ).

\section{Les Farguettes à Nissan-LeZ-EnsÉrune}

Dans la villa proche de l'oppidum d'Ensérune, les équipements vinicoles, attestés depuis le Haut-Empire, sont encore en fonction au $V^{e} s$. (Pellecuer, in: Garmy dir., 1996, p. 114-115). Dans son état initial, le chai, qui n'a été dégagé que sur une faible superficie, se présente comme une construction de plan rectangulaire d'environ $11 \mathrm{~m}$ de large et d'au moins $13 \mathrm{~m}$ de long. L'édifice est bâti dans le sens de la pente et la salle des pressoirs occupe naturellement le niveau le plus haut. La pièce, d'une surface de $50 \mathrm{~m}^{2}$, est dotée d'un puissant radier de plusieurs niveaux de blocs, qui devait être recouvert à l'origine d'une chape de béton. Deux bases maçonnées portent les jumelles des machines à levier. Le jus de presse s'écoule par gravité jusqu'à une cuve de 30 à $60 \mathrm{hl}$. L'agencement de l'espace consacré au stockage, situé au niveau le plus bas, est inconnu. On peut supposer que le bâtiment s'étend sur quelques dizaines de mètres et présente une forme allongée, qui est celle de la plupart des magasins à dolia.

Durant l'Antiquité tardive, d'autres aménagements traduisent l'apparition d'un nouveau circuit dans les étapes de la vinification. Les équipements de foulage et de pressurage sont nettement distincts, alors qu'antérieurement la salle des pressoirs devait servir à ces différents usages. Au Ves., une chape est coulée dans l'ancienne cuve de collecte du moût qui est ainsi transformée en fouloir. Un tesson de céramique paléochrétienne décorée (D-S-P) est mêlé aux éclats de tuiles du béton. On utilise en même temps une cuve, construite à cette époque ou lors d'une transformation antérieure du bâtiment. Elle a de façon approximative les mêmes capacités que celle du Haut-Empire. Elle peut servir à recueillir le liquide issu du foulage ou bien celui provenant d'un pressoir encore en fonction au niveau supérieur. L'activité vinicole ne disparaît que tardivement, au cours des $\mathrm{VI}^{\mathrm{e}}$ et $\mathrm{VII}^{\mathrm{c}} \mathrm{s}$., lorsque le chai est transformé en habitation ou en atelier, comme en témoigne la présence de foyers.

\section{Les JuRiètres-Basses à Puissalicon}

Pour la période du Haut-Empire, le site a livré les restes d'une installation de pressurage dont ne subsistent qu'un puissant radier et trois cuves. Les capacités de stockage sont estimées entre 195 et $235 \mathrm{hl}$, avec seulement une douzaine de dolia (Mauné et al., 1998, p. 79-87). Le caractère vinicole de cet ensemble particulièrement modeste est simplement une hypothèse. Les bâtiments sont abandonnés dans la seconde moitié du II ${ }^{e}$ s. et le site fait l'objet d'une réoccupation à partir de la fin du IV ${ }^{\mathrm{e}} \mathrm{s}$., et cela jusqu'au $V^{\circ} \mathrm{s}$. Elle se limite sur la surface fouillée à quelques fossés et fosses de fonction indéterminée. L'analyse anthracologique a montré que les dépôts du Ve s. se caractérisent par une représentation élevée de vitis vinifera. Selon L. Chabal (in: Mauné et al., 1998, p. 109), près de $12 \%$ de ce taxon est un taux suffisamment remarquable pour envisager avec quelque certitude la culture de la vigne alentour, avec récupération des résidus de taille pour le bois de feu. On ne connaît cependant aucun dispositif lié à la vinification pour la période considérée.

\section{Les Prés-Bas et Le Bourbou/ Port-De-Loupian à Loupian}

La villa des Prés-Bas, située sur la rive septentrionale de l'étang de Thau, probablement aux confins orientaux 


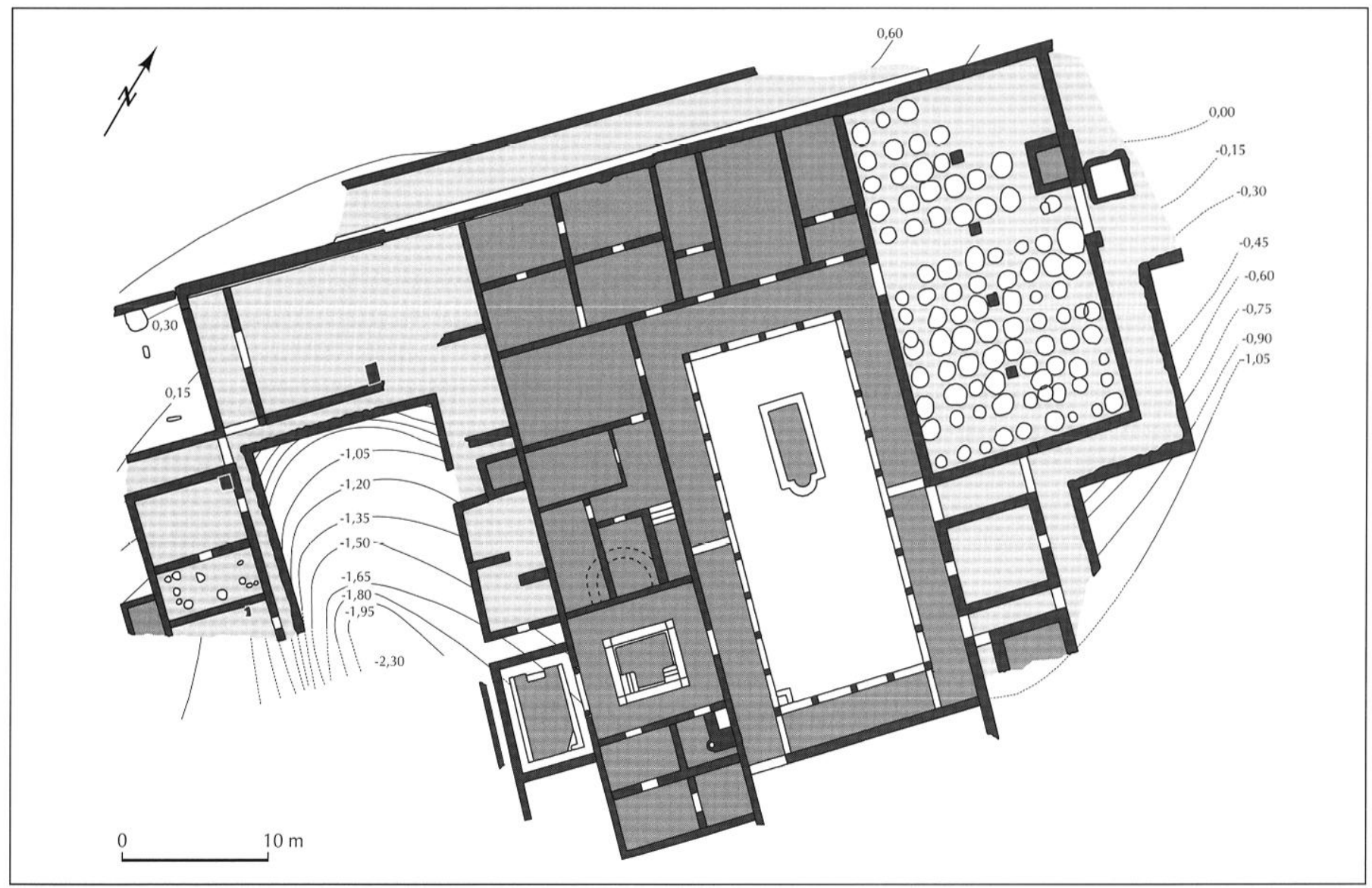

Fig. 44 - Un centre domanial de moyenne importance : la villa des Prés-Bas à Loupian (Hérault) (dessin C. Pellecuer).

de la cité biterroise, fait l'objet de fouilles depuis les années soixante. Cependant, les résultats présentés ici sont issus d'une série d'opérations programmées conduites sans interruption depuis 1983 sur le centre domanial et son territoire.

L'établissement rural est fondé au plus tôt vers le milieu du I ${ }^{\text {er }} \mathbf{s}$. avant notre ère mais ne prend l'aspect d'une villa qu'une centaine d'années plus tard. À la charnière du $\mathrm{I}^{\mathrm{er}}$ et du $\mathrm{II}^{\mathrm{e}} \mathrm{s}$. après J.-C., l'ensemble tel qu'il nous est connu (fig. 44) se présente sous la forme de trois corps de bâtiment disposés autour de trois espaces ouverts (Lugand et al., 1994, p. 251-253; Bermond, Pellecuer, 1998, p. 62). La partie centrale est occupée par le secteur résidentiel et sa cour à péristyle. On trouve à l'ouest des logements pour le personnel, à l'est les installations de production, chacune de ces parties donnant sur une cour basse. La dénivellation observée entre ces différentes composantes est due à un jeu de terrasses dont la mise en place s'est avérée nécessaire pour adapter les constructions à la topographie particulière du versant. C'est sur la terrasse la plus haute, à quelques dizaines de mètres seulement des bâtiments, qu'ont pu être repérés des aménagements agraires - fossés et petites fosses allongées - interprétés comme les traces de plantation d'un vignoble. Autant que l'on puisse en juger à partir des sondages réalisés, l'écartement moyen des creusements, de l'ordre de $1,7 \mathrm{~m}$ à $2 \mathrm{~m}$, laisse penser à un quartier de cultures spécialisées plutôt qu'à des cultures complantées.

Le chai est un bâtiment d'une certaine ampleur $\left(315 \mathrm{~m}^{2}\right)$, doté de deux nefs séparées par une file axiale de piliers. Les dolia, environ 90, sont disposés en deux travées d'importance inégale - deux tiers et un tiers, séparées par un espace de circulation. On a supposé que le remplissage des vaisseaux pouvait se faire au moyen de tuyauteries - peut-être même à l'aide d'une pompe - à partir d'une cuve d'un type particulier (compartiments et cuvelage en plomb ?) logée avec les récipients. Le 
ou les pressoirs doivent être localisés à l'extérieur du cellier. Là se trouve une fosse rectangulaire aux parois parementées qui a pu servir à la manœuvre d'un contrepoids. La capacité de stockage du chai est estimée à $1500 \mathrm{hl} \pm 250$; approximation qu'imposent nos incertitudes sur la contenance moyenne des dolia. En utilisant les rendements estimés par A. Tchernia (1995b) pour les vignobles d'époque romaine - entre 30 et $60 \mathrm{hl} / \mathrm{ha}$-, la surface plantée oscillerait de 10 à 50 ha, en tenant compte de la possibilité de conserver jusqu'à deux vendanges dans le cellier (Pellecuer, 1995, p. 190-191). Des performances inférieures, de moins de $20 \mathrm{hl} / \mathrm{ha}$, comme pour la période de la fin du Moyen Âge et l'époque moderne, sont certes possibles mais ouvrent sur des situations extrêmes où la superficie du vignoble peut avoisiner une centaine d'hectares. La vigne, on le sait, est une culture grande consommatrice de main-d'œuvre: l'exploitation de telles surfaces est-elle encore compatible avec la conduite d'une polyculture qui devait être la règle dans les domaines moyens du territoire biterrois ?

Le conditionnement de la production domaniale est assuré grâce aux amphores fabriquées dans un atelier distant d'à peine $1 \mathrm{~km}$, sur le rivage de l'étang de Thau (Bermond, Pellecuer, 1998, p. 63; Lugand, in: Garmy dir., 1999, p. 104-105). Les Gauloise 4 fabriquées au lieudit Le Bourbou ou Port-de-Loupian portent pour une partie d'entre elles - une sur quatre dans les tessonnières étudiées - la même estampille très stéréotypée, appliquée sur l'anse ou le col. La marque MAF, connue à ce jour à plus de 100 exemplaires dans l'atelier (Bermond, Pellecuer, 1998, p. 63), peut être interprétée comme les initiales des tria nomina du propriétaire. On a tenté, malgré les dangers d'une telle entreprise, de fixer un ordre de grandeur pour le nombre d'amphores fabriquées à partir de l'estimation des volumes de rebuts. Pendant une à deux générations, 40000 à 100000 conteneurs ont pu être défournés. On rappellera que le conditionnement d'une récolte stockée dans le chai de la villa nécessite un effectif d'environ 4000 amphores Gauloise 4 (Pellecuer, 2000, p. 246-248). On signalera enfin que plusieurs de ces récipients à la marque MAF, encore poissés, ont été retrouvés dans l'étang de Thau (Bermond, in: Garmy dir., 1999, p. 106).

L'activité globale de l'atelier court certainement sur une durée d'environ un siècle, à partir du dernier quart du $\mathrm{I}^{\mathrm{cr}} \mathrm{s}$. et jusque dans le courant du $\mathrm{II}^{\mathrm{c}} \mathrm{s}$. de notre ère. On s'appuie ici sur la chronologie établie à partir des céramiques d'origine non locale retrouvées dans les dépotoirs de production mais aussi sur la courbe de représentation des amphores gauloises dans les contextes du site des Prés-Bas. L'arrêt de la fabrication des conteneurs coinciderait avec d'importants remaniements affectant le chai de la villa. À cette époque, le nombre des dolia est réduit pour faire place à d'autres formes de stockage. Des bases maçonnées de forme rectangulaire $(1,2 \mathrm{~m}$ à $1,33 \mathrm{~m}$ de longueur pour $0,58 \mathrm{~m}$ à $0,7 \mathrm{~m}$ de large) sont espacées au maximum de $2,65 \mathrm{~m}$. Elles sont disposées en deux files distantes de $3 \mathrm{~m}$ environ. Serventelles à supporter de grands tonneaux de quelques dizaines d'hectolitres ? La découverte de céréales carbonisées autour de ces bases est-elle l'indice de la diversité des récoltes stockées dans l'ancien chai ? À n'en pas douter, de profondes transformations ont affecté l'organisation du domaine viticole du Haut-Empire (Pellecuer, 1996, p. 282-285).

Dans la seconde moitié du $\mathrm{IV}^{\mathrm{e}} \mathbf{s}$., un nouveau chai est construit à l'emplacement du magasin à dolia du HautEmpire (Pellecuer, 2000, p. 153-156). C'est un bâtiment d'environ $267 \mathrm{~m}^{2}$, de plan rectangulaire, dont le grand axe est perpendiculaire à celui du premier cellier. Malgré une réelle parenté dans les surfaces bâties des deux périodes, les capacités de stockage du nouveau bâtiment sont réduites de façon non négligeable à cause de la présence des installations de pressurage. Ces dernières, situées à l'extrémité ouest du bâtiment, contre le mur qui ferme l'aile résidentielle, occupent une surface de $60 \mathrm{~m}^{2}$ au maximum. Le jus de presse est recueilli dans une cuve, de 3,59 m de longueur sur 1,81 m, située à l'extérieur de la pièce de la machine. La cupule de décantation est placée au plus près de l'emmarchement, bâti dans un des angles. La cuve est directement accessible depuis l'espace de stockage. Une seconde cuve, de 4,11 $\mathrm{m}$ de longueur sur $1,53 \mathrm{~m}$ de large, est installée contre la salle de manneuvre, dans le prolongement de la salle du pressoir. Il n'y a aucun emmarchement et la cupule de décantation se trouve au centre de la cuve. Elle est peut-être utilisée en complément de la première, lors du pressurage, ou bien est-elle destinée plus spécifiquement à l'étape du foulage? Un espace d'environ $200 \mathrm{~m}^{2}$ est destiné au stockage du vin. Les contenants utilisés n'ont pas laissé de traces évidentes dans la surface qui a pu être fouillée. Quatre fosses à peu près circulaires, d'un diamètre inférieur à 1 m, ont été creusées dans le cellier, sans que l'on puisse déceler une quelconque disposition 
régulière. Compte tenu de la restitution proposée pour les niveaux de sol, l'arasement des vestiges n'est pas nécessairement la cause de la disparition des dolia et de leurs logements. Ce ne serait donc pas des récipients de terre cuite qui constitueraient l'essentiel des moyens de stockage du chai et l'hypothèse des tonneaux s'impose, si l'on veut tenter d'estimer les capacités du magasin. Selon les modules et le type de rangement utilisés, ce sont entre 300 et $800 \mathrm{hl}$ qui auraient pu être stockés.

Le site littoral du Bourbou où était installée la fabrique d'amphores du Haut-Empire est réinvesti à la fin du $\mathrm{IV}^{\mathrm{e}} \mathrm{s}$., ou plutôt dans le premier quart du $\mathrm{V}^{\mathrm{e}} \mathrm{s}$. (Pellecuer, 2000, p. 234-240). Trois cuves maçonnées du type bien connu dans les installations oléi-vinicoles ont été découvertes en deux points distincts. Les deux premières sont localisées à proximité du rivage. Elles ont été construites de façon successive au même emplacement. Il est à noter que leurs dimensions et aménagements respectifs - par exemple l'emmarchement placé à l'intérieur du cuveau - sont identiques. Le volume de la cuve la plus complète pouvait avoisiner $15 \mathrm{hl}$. La troisième est localisée à plus grande distance du rivage. Bien conservée sur une profondeur de $0,70 \mathrm{~m}$ environ, elle présente une forme rectangulaire et mesure environ $1,10 \mathrm{~m}$ sur $1,40 \mathrm{~m}$ de côté, soit un volume utile proche de celui des premières cuves. Elle est installée à l'intérieur d'un bâtiment quelque peu composite : une moitié est construite sur un solin de pierres liées à la terre. Cette partie abrite une recharge compactée faite d'un limon argileux mêlé d'éclats de tuiles. Dans l'hypothèse d'une installation vinicole, un fouloir, plutôt qu'un pressoir, occuperait cet espace d'une dizaine de mètres carrés. L'autre moitié, celle où est située la cuve pour recueillir le moût, semble plus ouverte que la première, protégée par un simple auvent sur poteaux. L'ensemble occupe une surface réduite d'environ $30 \mathrm{~m}^{2}$ hors œuvre. Un tel bâtiment semble bien suffisant pour faire face au traitement des petites quantités récoltées.

\section{La Quintarié à Clermont-l'Hérault}

Dans le cadre des travaux archéologiques préalables à la réalisation de l'autoroute $\mathrm{A} 75$, fouilles et opérations d'évaluation sur une surface de 25 ha permettent de cerner l'évolution d'un établissement rural d'un type original et du vignoble qui l'entoure (Pomarèdes dir., 1999).
Les constructions du Haut-Empire, que l'on date des années 75-140 après J.-C., sont installées au croisement de deux chemins. L'un d'eux pourrait correspondre à un axe de première importance qui relierait Cessero (Saint-Thibéry, Hérault), sur le tracé de la voie Domitienne, Luteva (Lodève, Hérault), et Condatomagus (Millau, Aveyron). L'établissement est composé de trois ensembles de constructions distincts, répartis sur $4500 \mathrm{~m}^{2}$ (fig. 45). Un petit complexe thermal, doté d'une abside, est construit au bord de la voie majeure. Plus au sud-est, s'élève un ensemble de trois bâtiments assez similaires. Enfin, au nord, un édifice regroupant l'ensemble des installations de production et deux petits corps de bâtiment latéraux sont implantés le long de la voie secondaire.

Les aménagements mis en évidence attestent la présence d'installations vinicoles dont les capacités de stockage sont assez limitées. Dans le chai, quatre cuves ont été découvertes. Construites à différents moments, elles seraient utilisées apparemment par paires. Un pressoir pourrait être localisé dans une salle établie en avant du cellier. En l'état des vestiges, on ne peut proposer qu'une prudente reconstitution. Deux puissantes bases maçonnées, chaînées aux murs latéraux, supporteraient un système de potence et de palan susceptible de relever le bras d'un pressoir à treuil. En l'absence de pressoir, c'est au moins un fouloir qu'il semble nécessaire de restituer dans cette salle. Enfin, une quarantaine de dolia ont été dégagés. Vingt-quatre à vingt-huit de ces vaisseaux ont été utilisés concurremment. Ils sont de contenance moyenne, entre 12 et $15 \mathrm{hl}$, ce qui permet d'évaluer la capacité de stockage des celliers dans une fourchette comprise entre 288 et $420 \mathrm{hl}$.

Si l'on suppose que les centaines de traces de plantation découvertes aux abords du site correspondent aux pratiques de repiquage ou de marcottage de plants de vigne, on peut considérer que le vignoble occupe une place prépondérante dans ce terroir. Un premier découpage parcellaire, en une douzaine de champs plantés en vignes, a pu être proposé. Ils ont été distingués et délimités grâce aux différences d'orientation des alignements de fosses et à l'extension de chaque groupe. On a pu observer en outre deux cas différents d'articulation entre les façades des bâtiments et le semis de plantation. Aucun cas de superposition n'a été remarqué et les datations apportées par le mobilier retrouvé dans 


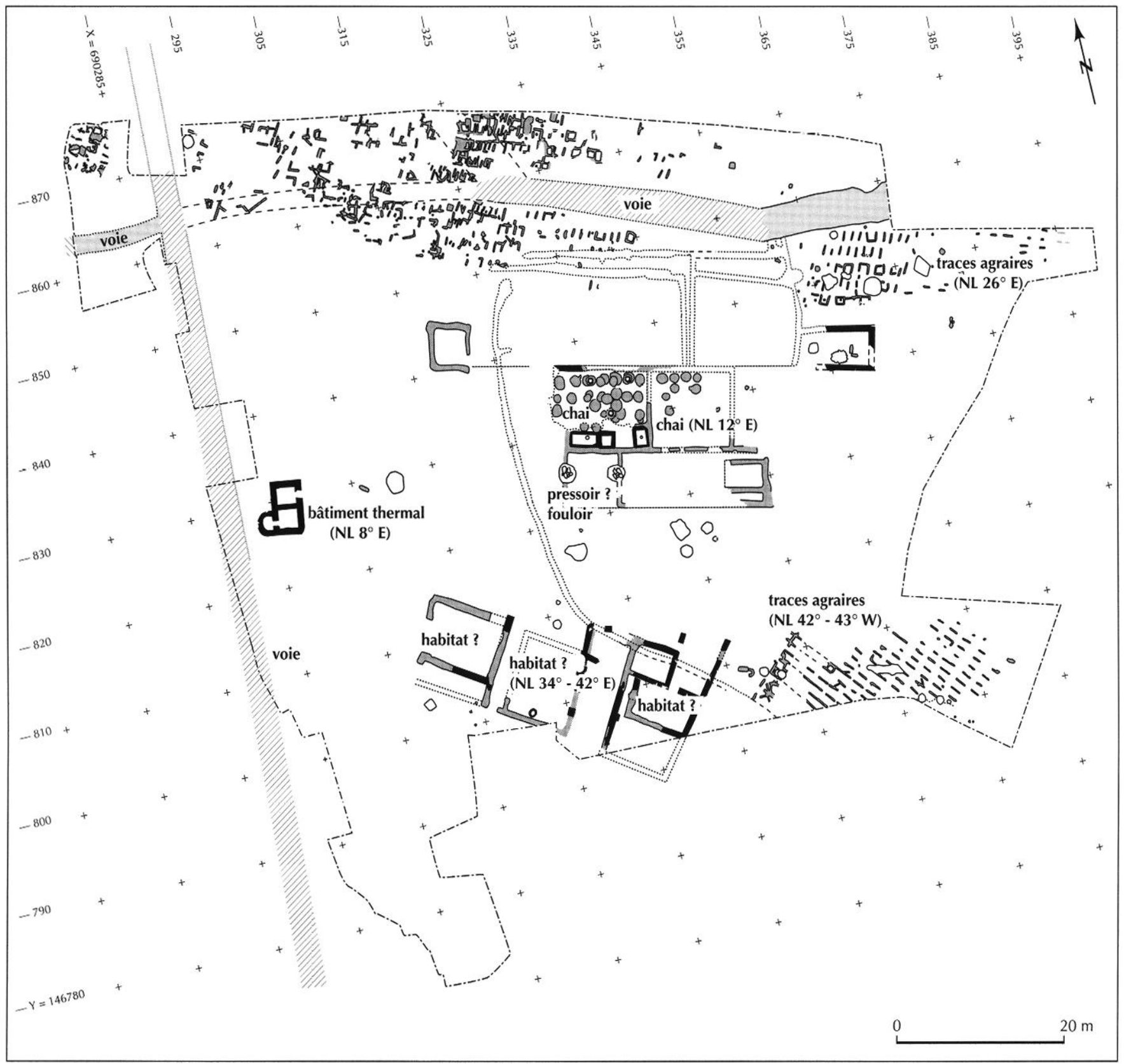

Fig. 45 - Élablissement viticole et vignoble de La Quintarié à Clermont-l'Hérault (Hérault) (dessin X. Chadefaux, L. Cordier, AFAN).

chaque fosse signent une mise en place du vignoble entre le $\mathrm{I}^{\text {er }}$ et le début $d u \mathrm{II}^{\mathrm{e}} \mathrm{s}$. après J.-C. Son extension est évaluée entre un et deux tiers de l'espace agricole potentiel. Des fosses d'un autre type, de section quadrangulaire, beaucoup plus espacées les unes des autres, ont été retrouvées concentrées dans deux à trois secteurs seulement, consacrés probablement à d'autres formes d'arboriculture. Les zones vides de traces peuvent correspondre à des cultures annuelles ou des pâturages.
Il n'est pas exclu cependant que quelques légères éminences aient été tronquées du fait de travaux agricoles postérieurs et que les vestiges du vignoble n'aient été conservés que dans les zones correspondant à de modestes dépressions.

L'échantillonnage dont nous disposons permet d'entrevoir un vignoble de grande étendue, gagné sur toutes les terres disponibles. Il a fallu s'adapter à la trame des voies, à une topographie assez sinueuse en pied de 
versant et à diverses orientations parcellaires, sûrement préexistantes, qui ont influencé les directions des champs et les bâtiments. Au vu de nos évaluations, soit 40 fosses pour $80 \mathrm{~m}^{2}$, près de 5000 pieds sont plantés à l'hectare ce qui correspond à une densité plutôt élevée. Pour les seuls 25 ha diagnostiqués, le nombre de pieds se situerait entre 40000 et 80000 selon la part des terres consacrées au vignoble. L'unité de production de La Quintarié, avec des capacités de stockage inférieures à $500 \mathrm{hl}$, ne peut être à même de maitriser de telles surfaces plantées. Une superficie en vignoble comprise entre 7 et 14 ha, en supposant un rendement à l'hectare relativement bas compris dans une moyenne de 30 à $40 \mathrm{hl}$, correspondrait aux possibilités de l'exploitation rurale. Il est à signaler que deux petits établissements ruraux, distants de 600 à $700 \mathrm{~m}$ et occupés à la même époque que le site fouillé - Les Garels à Brignac et Fouscaiis à Clermont-l'Hérault (Schneider, Garcia, 1998, p. 144 et 165) - ont pu être été impliqués dans la mise en valeur de ce vaste vignoble.

H. P.

\section{Varemles à Paulhan/Adissan}

Découverte à la fin des années 1970 à l'occasion de travaux agricoles, la villa de Vareilles ${ }^{132}$ correspond à un très grand établissement rural occupé entre la première moitié $d u I^{\text {er }}$ s. après J.-C. et le courant du III $^{e}$ s. À la charnière du I ${ }^{\text {er }}$ et du II $^{e}$ s., on peut restituer une villa étendue, d'une superficie supérieure à 1,2 ha, associant de très grandes installations agricoles (équipements de transformation et de stockage, moulins hydrauliques) et artisanales (métallurgie) ainsi qu'un vaste secteur résidentiel dont on a bien appréhendé l'aile thermale.

Les corps de bâtiment du secteur agricole s'organisent en partie autour d'une vaste cour centrale de $1200 \mathrm{~m}^{2}$ (fig. 46). Les aménagements relevant d'une activité viticole sont nombreux mais sont conservés de façon très inégale. Les installations de foulage et de pressurage ont beaucoup souffert des travaux agricoles. Ainsi, la salle des pressoirs n'a conservé qu'une faible partie de

132. Situé à l'emplacement d'un échangeur de la future A75, le site fait l'objet d'une fouille extensive sur près de 2 ha depuis le mois de juin 1999. Le premier bilan de ces travaux qui est proposé ici (oct. 1999) doit être considéré comme provisoire. La fouille est conduite avec la collaboration de F. Cavalin, J. Planchon, Z. Sekhari et P. Vidal (AFAN). son remblai de galets et seulement la semelle de fondation de deux cuves ${ }^{133}$. Les chais sont en revanche bien moins dégradés et nous renseignent sans ambiguité sur la place prépondérante occupée par la viticulture au sein des activités de ce grand domaine. Le premier, très arasé, se trouve rejeté à l'extérieur de l'ensemble organisé autour de la cour. Il prend place dans une zone où l'on a reconnu des tranchées de plantation de vignes de la première moitié du $\mathrm{I}^{\text {cr }} \mathrm{s}$. de notre ère. Seuls les fonds des dolia ont subsisté et aucun mur n'a été épargné par les travaux agricoles. Le cellier n'a connu qu'une seule période d'utilisation. Le deuxième espace réservé au stockage se situe à peu de distance du précédent, dans l'une des salles de l'aile méridionale du grand bâtiment à cour centrale. Malgré l'arasement, on peut estimer la capacité du chai à une cinquantaine de dolia. Ceux-ci présentent des morphologies et des pâtes hétérogènes: peut-être s'agit-il d'une salle de stockage conçue pour faire face à une très bonne récolte. En tenant compte des volumes moyens attribués à ces récipients ( 12 à $18 \mathrm{hl})$, les capacités de stockage de chacun des deux ensembles décrits doivent être respectivement fixées entre 360 et $450 \mathrm{hl}$ et entre 600 et $900 \mathrm{hl}$.

Un troisième chai, dans l'angle est de la cour, a livré les restes de 27 dolia, de même origine et dont le volume global peut être fixé entre 324 et $486 \mathrm{hl}$. Fnfin, dans toute. la moitié nord du grand complexe à cour centrale, l'espace en " $L$ " est entièrement réservé à un immense chai contenant au moins 310 dolia qui sont de grande contenance - entre 15 et $18 \mathrm{hl}$ - et ont tous la même origine. Une monnaie trouvée dans le grand mur de façade occidental place la construction de cet ensemble, certainement réalisé en un seul jet, après les années 40 de notre ère. Les capacités de stockage de ce dernier ensemble sont très importantes, de l'ordre de 4650 à $5580 \mathrm{hl}$ pour plus de 250 dolia. Si l'on envisage - comme nous y invitent les données chronologiques fournies par la fouille - que les quatre chais aient pu être utilisés dans la première moitié du $\mathrm{II}^{\mathrm{e}} \mathrm{s}$., le total atteindrait 5930 à $7400 \mathrm{hl}$. Si cette hypothèse était avérée, on serait en présence de l'un des plus grands établissements vinicoles connus à ce jour en Narbonnaise. À titre de comparaison,

133. D'autres cuves ont été découvertes à distance des bâtiments agricoles réunis autour de la cour. En l'état d'avancement de la fouille, on se gardera de se prononcer sur leur fonction. Elles sont en effet situées à proximité d'installations artisanales (tissage, métallurgie, etc.). 


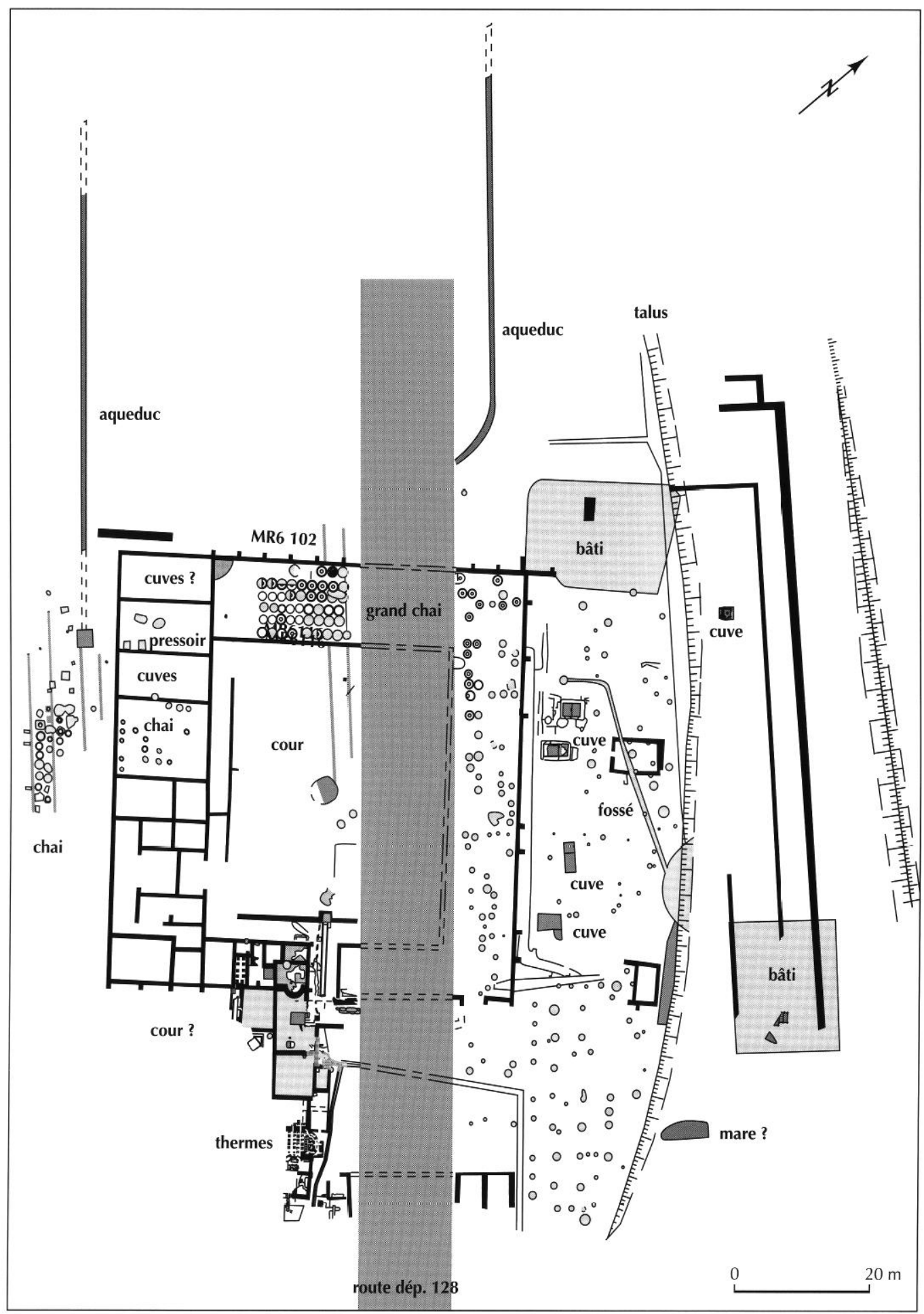

Fig. 46 - Un exemple de grand établissement viticole spécialisé : Vareilles à Paulhan/Adissan (Hérault) (dessin F. Rebière, AFAN).

la villa du Molard à Donzère (Drôme), considérée jusquelà comme le type même de la très grande exploitation viticole, dispose d'une capacité de $2500 \mathrm{hl}$ (Odiot, 1996).

Bien que ces chiffres demandent encore à être discutés, on doit convenir de toute évidence que la pro- duction viticole du domaine de Vareilles et donc la surface plantée en vignes étaient très importantes. En ne tenant compte que du chai en " $\mathrm{L}$ » et même si on utilise des chiffres moyens $-15 \mathrm{hl}$ pour les dolia et $30 \mathrm{hl} / \mathrm{ha}$ de rendement, le vignoble pouvait dépasser 120 ha, ce qui 


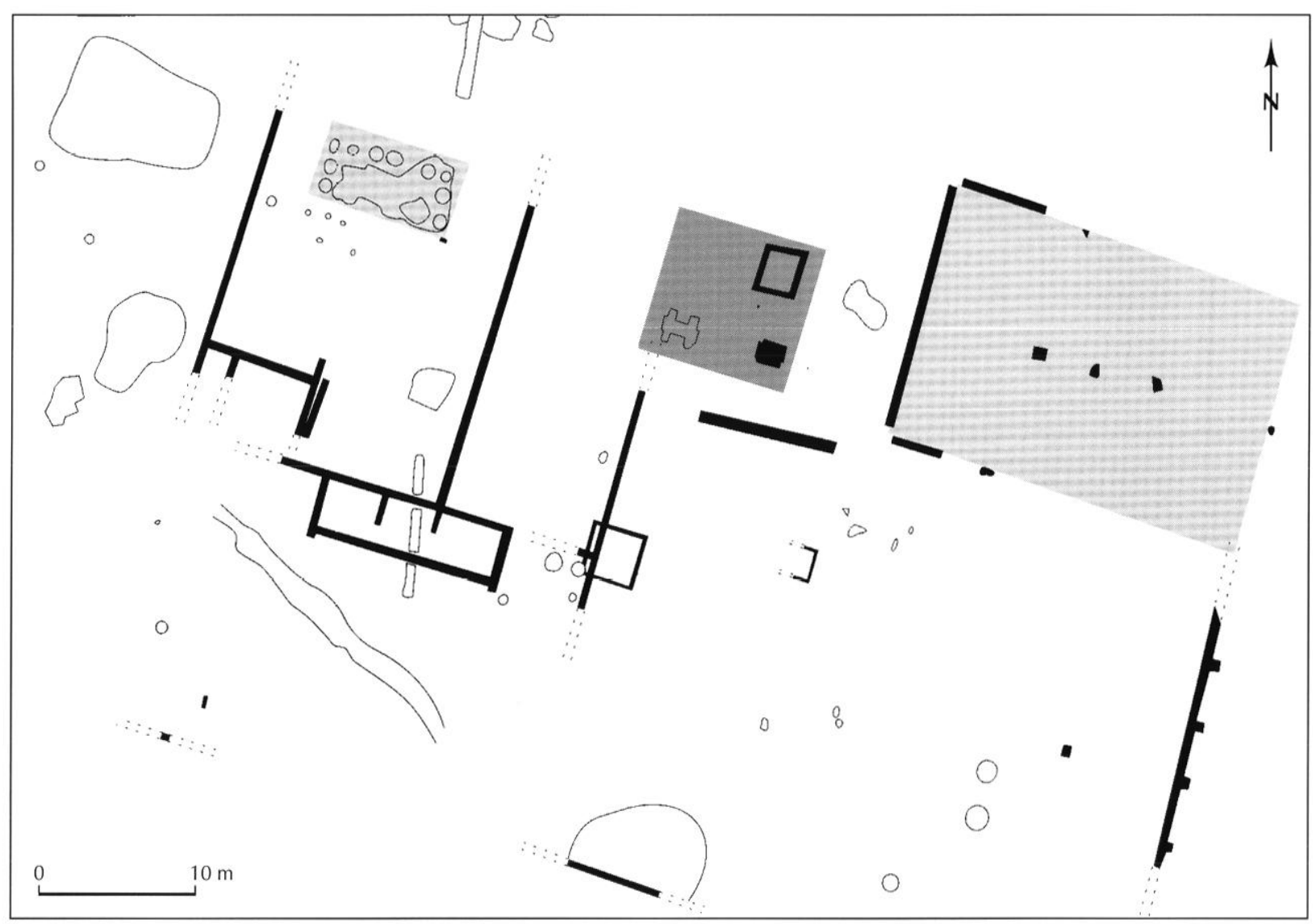

Fig. 47 - La villa du Petit Clos à Perpignan, Hérault : en grisé clair, les différents espaces de stockage; en grisé sombre, les pressoirs (dessin P. Garczynski, CEPAM-CNRS d'après J. Kotarba, complété).

est considérable. Cette villa constitue probablement le principal site viticole de la moyenne vallée de l'Hérault. Il est à noter qu'elle s'insère d'ailleurs dans un réseau dense et hétérogène d'établissements ruraux également tournés vers cette activité agricole (Mauné, 1998, p. 193-201; Mauné, 2001a). Dans cette zone privilégiée, la forte densité des officines produisant, entre autres choses, des amphores Gauloise 4 confirme également la part prise par une viticulture de type spéculatif (Laubenheimer, 1985 ; Ginouvez, Mauné, 1996 ; Mauné, 2001a).

S. M.

\section{DÉPARTEMENT DES PYRÉNÉES-ORIENTALES}

\section{Le Petit Clos à Perpignan}

La villa du Petit Clos a fait l'objet de campagnes de fouilles successives au fur et à mesure de la réalisation d'un lotissement de la périphérie de Perpignan (Kotarba, 1996). Du fait d'un arasement important du site, le plan de l'ensemble est très lacunaire et l'on distingue plusieurs types d'aménagement qui peuvent être mis en relation avec une activité viticole (fig. 47). Deux aires de stockage de faible ampleur, composées de 18 et 24 dolia chacune (environ $600 \mathrm{hl}$ ), sont placées au centre d'une cour et protégées par une toiture de tuiles supportée par des poteaux. À proximité, s'élève un édifice à deux nefs, de $15 \mathrm{~m}$ de large pour une longueur comprise entre $16 \mathrm{~m}$ et $22 \mathrm{~m}$. Bien qu'aucun dolium ou logement de récipient n'ait été retrouvé à l'intérieur, il pourrait bien s'agir d'un chai. Son plan est caractéristique avec sa file axiale de piliers et ses capacités, de l'ordre de $300 \mathrm{~m}^{2}$, conviendraient à une exploitation viticole qui dispose d'une production de $1000 \mathrm{hl}$. En outre, à moins de $10 \mathrm{~m}$ de la façade orientale du bâtiment, se trouve une cuve en opus spicatum. De forme rectangulaire $(2,10 \mathrm{~m}$ sur $2,60 \mathrm{~m})$, elle présente en son 
centre une légère dépression. Elle est accompagnée d'un massif maçonné quadrangulaire et d'un assemblage de pièces de bois, dont on a retrouvé le négatif en forme de « $\mathrm{H}$ » dans le sol naturel. On aurait là la base d'un pressoir à vis. Un atelier de potiers, en contrebas du centre domanial, a produit des tuiles et de la vaisselle mais peut-être aussi les amphores nécessaires au conditionnement du vin.

\section{Pla de l'Aïgo à Caramany}

Cette ferme, occupée depuis la fin du $\mathrm{I}^{\mathrm{er}} \mathrm{s}$. de notre ère, a pu être fouillée préalablement aux travaux de mise en eau du barrage de l'Agly. Pour l'état qui est daté au plus tôt du milieu du IIIc $\mathbf{s}$., on signale la construction de ce qui semble bien être un pressoir. Le sol incliné de la pièce de $3,65 \mathrm{~m}$ de large sur $5 \mathrm{~m}$ de long reposait sur un double hérisson de galets disposés de chant et noyés pour leur partie supérieure dans un mortier de chaux de bonne qualité. La salle n'est séparée de la pièce moyenne que par une sorte de marche dont une face est enduite d'un béton de tuileau. Aucune cuve ou structure de stockage n'est signalée à proximité (Fabre et al., 1999, p. 275-276).

\section{LA VIGNE ET LA PRODUCTION DE VIN DANS LA PARTIE OCCIDENTALE DE LA NARBONNAISE}

\section{LA VITICULTURE RÉGIONALE, DE LA PÉRIODE DE LA CONQUÊTE AU MILIEU DU I ${ }^{\text {er }}$ S. APRÈS J.C.}

Récemment encore, l'émergence d'une viticulture locale dans cette partie d'une Transalpine fraîchement conquise n'était établie qu'en contrepoint de la courbe descendante des importations d'amphores vinaires d'Italie puis de Tarraconaise. Cette tendance, mise en évidence à partir des sites de consommation, était confirmée par le démarrage, autour du changement d'ère, de la fabrication régionale de conteneurs. Les ateliers de cette première génération - Mauressip à SaintCôme-et-Maruéjols, Gard (Laubenheimer, 1985, p. 149-152 et 385), Îlot Thérond à Nîmes, Gard (Monteil, 1999b, p. 246-249), Saint-Bézard à Aspiran, Hérault, La Teularié à Corneilhan, Hérault (Laubenheimer, 1985, p. 385-386), peut-être Font del Mas à Ponteilla, Pyrénées-
Orientales (Olive, Pezin, 1997) - sont répartis en divers points de la région languedocienne. Les preuves d'une culture de la vigne au cours de l'âge du Fer et la pratique de la vinification par les sociétés indigènes sont des éléments nouvellement établis, qui ont considérablement nuancé cette approche ( $c f$. Py et Buxó y Capdevila, supra, p. 29-43). Les premières fabriques d'amphores constituent, à n'en pas douter, des témoignages incontestables des progrès de la viticulture dans les cités de la Narbonnaise occidentale. Elles apparaissent aujourd'hui comme les indicateurs les plus tangibles d'une nouvelle étape dans les changements apportés aux conditions de production et de commercialisation du vin régional $d u$ fait de la romanisation ${ }^{134}$.

C'est à Nîmes (Gard) qu'a été retrouvé l'un des plus anciens vignobles connus à ce jour. Sa création est située dans le dernier quart du $\mathrm{II}^{\mathrm{e}} \mathrm{s}$. avant notre ère (Monteil, in: Garmy dir., 1999, p. 69 ; Monteil et al., 1999). Deux champs, dont la superficie exacte reste inconnue, ont livré des fosses de plantation dont le rythme serré indique un quartier de cultures spécialisées. Les terres sont localisées à moins de $200 \mathrm{~m}$ au sud de la limite méridionale de la ville préaugustéenne. La période est marquée par une très forte expansion de l'habitat, signe d'une croissance démographique et d'un net développement économique (Monteil, 1999b, p. 324-327). À cette même date, on connaît dans la proche périphérie de l'agglomération des travaux de génie rural d'une importance certaine, comme les terrasses et les champs irrigués découverts lors de la fouille de la ZAC des Halles (Monteil dir., 1993, p. 29-79). À partir de ce tableau à peine ébauché, on a tenté de cerner les promoteurs de tels investissements. L'hypothèse de colons italiques repose sur l'idée d'une rupture, culturelle et technique, avec l'agriculture des sociétés locales (Poupet, in: Monteil dir., 1993, p. $73-75)^{135}$. On peut aussi penser, avec M. Monteil, que ces aménagements agraires - création de vignobles et. nouvel agencement des champs - sont à l'initiative de riches notables indigènes (Monteil, 1999b, p. 491 et 493 ; Monteil et al., 1999, p. 116-117). La romanisation aurait

134. Pour compléter ce dossier, on signalera la découverte récente à Marscillan (Hérault), à proximité d'Agde et des rives de l'étang de Thau, des restes d'un atelier qui aurait fabriqué des amphores de type Dressel 1 et qu'il faudrait associer à une installation viticole du $I^{\text {er }} s$. avant notre ère (fouilles $\mathrm{E}$. Gome\%, 1999, inédit).

135. Hypothèse admise par M. Py et R. Buxó i Capdevila. 
en effet permis l'apparition d'une nouvelle paysannerie, étroitement dépendante d'une aristocratie locale qui fournira l'essentiel des élites et des grands propriétaires fonciers du Haut-Empire (sur ces questions, voir Fiches, 1989a et 1993).

En Roussillon, des prospections menées de façon intensive depuis plusieurs années ainsi que des fouilles permettent de mieux cerner les formes prises par l'habitat rural dans la proche campagne de la colonie latine de Ruscino/Château-Roussillon à Perpignan, Pyrénées-Orientales (Comps, Kotarba, 1997). Dans huit cas au moins, des bassins et des cuves dont le fond est constitué de briquettes disposées en épi ont été signalés. Les fonctions de ces aménagements semblent diverses, certains d'entre eux ayant pu servir de citernes. Au Mas Coste/Palais des Sports à Perpignan, l'une de ces constructions est datée des années 20-40 de notre ère (Kotarba, in: Massy dir., 1993, p. 105-106). Dans la villa du Petit Clos à Perpignan, la cuve en opus spicatum est en relation avec un pressoir. En l'état des recherches, l'hypothèse d'installations vinicoles dans la plaine du Roussillon dès la première moitié du $\mathrm{I}^{\mathrm{er}} \mathrm{s}$. de notre ère est envisagée ${ }^{136}$.

À partir du deuxième quart du I ${ }^{\mathrm{er}}$ s. après J.-C., l'augmentation du nombre des amphores gauloises sur les sites de consommation de la partie occidentale de la Narbonnaise irait de pair avec un accroissement de la production régionale (Laubenheimer, in: Fiches, 1989b, p. 121-128; Laubenheimer, 1985, p. 389). Dans la basse vallée du Rhône, trois ateliers autour de Bagnols-sur-Cèze (Gard) ont tourné des amphores Gauloise 1, d'un type connu depuis la fin de la période augustéenne. Ces récipients, dont la pâte sableuse est très caractéristique (Laubenheimer, 1985, p. 128, 135-140 et 161-166), sont représentés dès le deuxième quart $d u \mathrm{I}^{\mathrm{er}} \mathrm{s}$. dans les contextes régionaux ${ }^{137}$. Cette même région bagnolaise a

136. Une cuve du même type que celle de la villa du Petit Clos a été découverte sur le site des Farguettes à Nissan-lez-Ensérune (Hérault), établissement rural pour lequel une notice a été donnée supra, p. 96. Cette découverte isolée en un secteur du gisement n'est malheureusement pas datée de façon précise.

137. Les travaux de S. Barberan (AFAN) sur les amphores gauloises précoces en Languedoc oriental amèneront sûrement à nuancer l'idée couramment admise d'une diffusion de l'amphore Gauloise 1 dans la première moitié du $\mathrm{I}^{\mathrm{er}} \mathrm{s}$. de notre ère. La forme « classique » ne semble apparaitre dans les contextes urbains nîmois qu'à partir du milieu du $\mathrm{I}^{\text {er }}$ s. après J.-C. fait l'objet de prospections systématiques qui ont permis de retrouver un dense semis de fermes et de villae ${ }^{138}$. Parmi ces dernières, la seule fouillée est la villa de La Ramière à Roquemaure (Gard). Dans la première moitié du I ${ }^{\mathrm{er}} \mathrm{s}$. de notre ère, on n'y trouve pas encore de chai doté de dolia ou même de pressoir. Deux pièces peuvent être interprétées comme des fouloirs et une sorte de chaudière aurait été utilisée pour la préparation du defrutum.

\section{LE DÉVELOPPEMENT SANS PRÉCÉDENT DU HAUT-EMPIRE}

L'acmé de la production viticole sud-gauloise est liée de façon indissociable à l'apparition d'une amphore destinée au grand commerce, la Gauloise 4 dont on a souligné le caractère très standardisé de la fabrication malgré la multiplicité des officines régionales (Laubenheimer, 1998a, p. 167). Sa diffusion commence à partir du milieu du I Irr $\mathrm{s}$. après J.-C. pour s'intensifier au cours du $\mathrm{II}^{\mathrm{e}} \mathrm{s}$., comme le montrent les taux de représentation de ce conteneur sur les lieux de consommation extra-gaulois (Ostie, Augst...). Comme dans l'ensemble de la province, cette période est marquée par une intense croissance de l'habitat rural en Narbonnaise occidentale (Archaeomedes, 1998, p. 75 sqq). Les villae se multiplient et plus de $90 \%$ d'entre elles sont occupées au cours de cet intervalle de temps (Pellecuer, Pomarèdes, 2001). L'ampleur des investissements se mesure de façon concrète avec le développement des fermes et des centres domaniaux de plan régulier, et le recours de plus en plus répandu à une construction à base de mortier de chaux. Le fléchissement n'est perceptible qu'à partir du $\mathrm{III}^{\mathrm{e}} \mathrm{s}$. avec une décrue très nette de l'effectif des sites ruraux et une baisse plus mesurée du nombre des centres domaniaux. On notera que la Gauloise 4 est fabriquée sans interruption durant tout ce siècle dans un atelier tel que celui de Sallèles-d'Aude, signe probant de la poursuite des exportations.

Durant le Haut-Empire, les efforts consentis par les propriétaires fonciers touchent au premier chef les équipements vinicoles dont les vestiges conservés peuvent, on le verra, être assez spectaculaires. Quatre-vingts de ces installations, le plus souvent incomplètement connues,

138. Travaux de H. Petitot ; sur les recherches dans la vallée de la Tave, voir en dernier lieu, Petitot, in: Garmy dir., 1999, p. 170. 


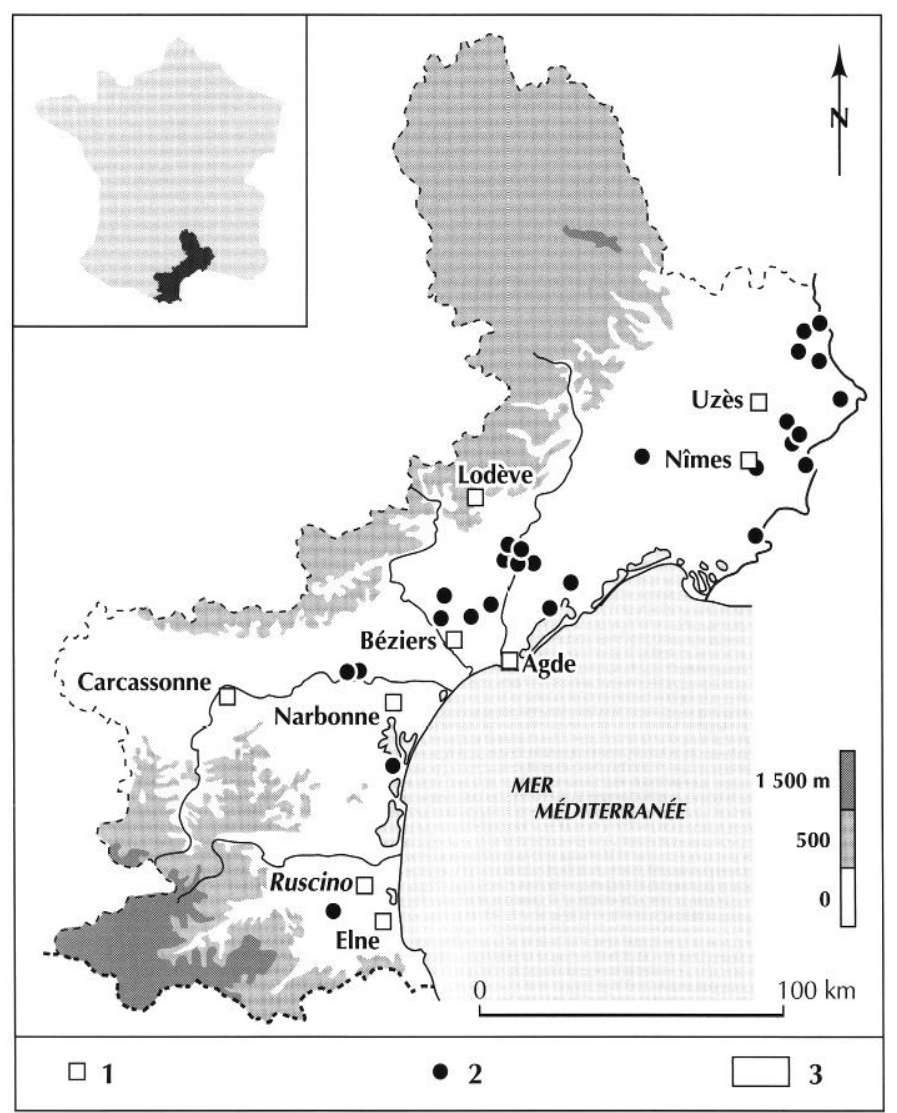

A

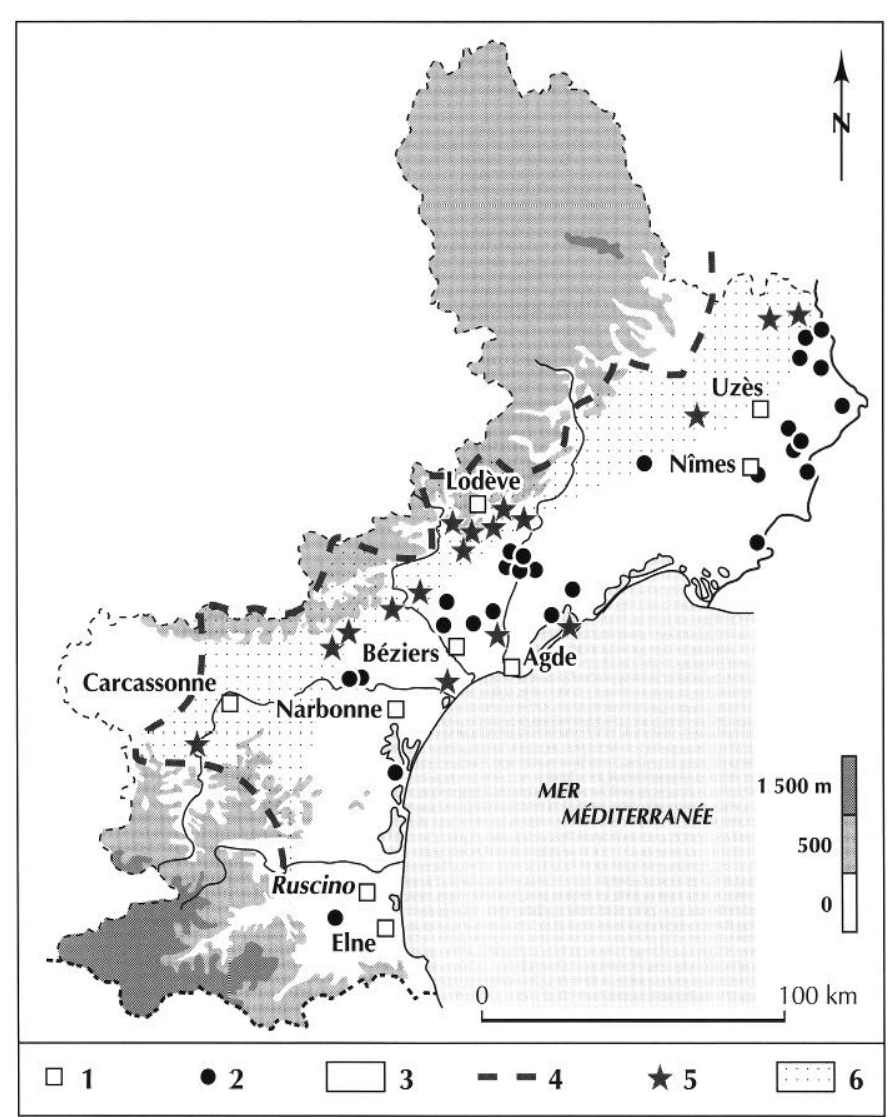

B

Fig. 48 - Géographie de la production viticole en Narbonnaise occidentale.

A. La distribution des vestiges pouvant relever d'installations viticoles est corrélée par celle des ateliers ayant produit des amphores gauloises; on peut ainsi distinguer trois zones particulièrement dynamiques : la plus imporlante correspond pour l'essentiel aux territoires des cités de Béziers et de Narbonne; la zone rhodanienne et une partie de la plaine roussillonnaise constituent deux autres secteurs remarquables mais de moindre étendue: 1, principales agglomérations gallo-romaines; 2 , ateliers d'amphores vinaires; 3 , secteur où la culture de la vigne est prédominante d'après la découverte d'installations de production.

B. La carte précédente apporle quelques nuances au zonage des cultures proposé par D. Garcia (1992a, p. 251, fig. 28), qui est marqué par l'opposition de la plaine viticole et des premiers reliefs consacrés à l'oléiculture : 1, principales agglomérations gallo-romaines; 2, ateliers d'amphores vinaires ; 3, zone à culture de la vigne prédominante ; 4, limite de la culture de l'olivier ; 5, huilerie ; 6, zone à culture de l'olivier prédominante (dessin C. Pellecuer, fond de carte C. Olive).

ont pu être recensées en Languedoc-Roussillon et se rapportent pour une très large majorité à la période du Haut-Empire ${ }^{139}$. Les départements de l'Aude et de l'Hérault, qui couvrent l'essentiel des cités antiques de Narbonne et de Béziers, ont livré chacun une trentaine d'occurrences alors que moins d'une quinzaine sont connues dans le Gard - une large partie de la cité de Nîmes - et les Pyrénées-Orientales. On ne peut nier le

139. Enquête en cours menée par Aline Avril à partir des données par la cellule "Carte archéologique nationale " du Service régional de l'archéologie du Languedoc-Roussillon. caractère encore partiel de cette enquête à cause du déséquilibre qui existe dans la documentation à notre disposition pour les différents départements. Autour de Narbonne et de Carcassonne, il faut se contenter d'une distribution bien clairsemée de découvertes ponctuelles que vient compléter le semis plus serré des officines connues. Seul le développement d'une archéologie préventive permettrait de donner plus de densité à la définition de cette zone viticole, qui peut paraître aujourd'hui bien surévaluée. Malgré cet état d'avancement inégal des recherches dans les divers secteurs de la Narbonnaise occidentale, une géographie de la production est en 
train de se dessiner dans une région que l'on a pu considérer dans un premier temps comme entièrement viticole (fig. 48) (Garcia, 1992a, p. 250-255). L'extension du vignoble pourrait toucher en priorité des secteurs de la plaine languedocienne " où les modelages et remodelages cadastraux ont été les plus anciens et les plus importants " (Laubenheimer, 1998b, p. 372).

\section{L'EXEMPLE DE LA CITÉ DE NÎMES}

Dans le territoire nîmois, ce sont les agglomérations secondaires qui ont plus particulièrement retenu l'attention des chercheurs ces dernières années. Or, ces sites n'ont livré qu'une quantité assez maigre de renseignements relatifs à la question viticole. Les établissements ruraux plus à même de nous renseigner n'ont été que rarement étudiés, mais l'on dispose tout de même d'observations ponctuelles et de deux sites de villae ayant fait l'objet de fouilles extensives (La Ramière à Roquemaure et Saint-André-de-Codols à Nîmes). D'un point de vue géographique, le recensement des ateliers montre une concentration en bordure du Rhône (fig. 49). C'est également dans le sillon rhodanien que les installations viticoles connues sont les plus abondantes, de la vallée de la Cèze au petit Rhône.

La région bagnolaise livre les éléments les plus nombreux. À Laudun (Gard), au pied de l'oppidum du Camp de César, agglomération occupée durant toute l'époque romaine, deux cuves jumelées sont connues dans la villa de Bas-Claud et un cellier à dolia, apparemment vaste, dans une autre villa située au sud du village actuel ${ }^{140}$. Parmi les nombreuses grandes villae recensées le long du Rhône, beaucoup devaient participer à cette production de masse. La seule fouillée, celle de La Ramière à Roquemaure (Gard), donne une image peu représentative de ce qu'était un domaine viticole dans ce secteur. Au cœur d'un ensemble bâti occupant plus de $1 \mathrm{ha}$, on ne trouve qu'une installation de stockage aux dimensions assez réduites: les deux celliers à dolia reconnus ne devaient contenir que 400 à $500 \mathrm{hl}$. En outre, il n'y a sur le site aucune trace nette de la présence d'un pressoir, à cause, il est vrai, du fort arasement qu'a connu le site. Au nord du Gardon, dans la vallée de

140. On compte également plusieurs sites ayant livré en prospection de vastes concentrations de fragments de dolia, marquant très certainement l'emplacement de celliers à vin.
l'Alzon, les indices sont plus rares. On connaît néanmoins sur la commune de Vers-Pont-du-Gard (Gard) un atelier d'amphores Gauloise 4 récemment découvert au lieu-dit Font de Dringues (Buffat, in: Garmy dir., 1999, p. 81-82) et une villa, Valagrand, qui a livré à l'occasion d'un sauvetage un bloc de jumelle de pressoir et un bassin, liés à une installation vinicole (Benoit et al., 1994, p. 161 ; Provost et al., 1999, p. 728, fig. 911). Autour de Beaucaire/Ugernum (Gard), les prospections ont révélé la présence de plusieurs grandes villae associées à un atelier d'amphores (Bessac et al., 1987, p. 106 ; Laubenheimer, 1989b, p. 11 ; Provost et al., 1999, p. 225 et 226).

À mesure que l'on s'éloigne du Rhône, les indices de production deviennent plus rares. Dans la périphérie de la ville antique d'Uzès/Ucetia (Gard), on ne connaît aucun atelier d'amphores, mais les campagnes de prospection ont été moins nombreuses dans ce secteur. De grandes villae sont connues. Un sondage sur celle des Auvis à Flaux a tout de même révélé une cuve de recueil (Ratz, in: Massy dir., 1994, p. 66), probablement en relation avec une production de vin.

La partie occidentale de la cité de Nîmes s'individualise par la minceur de la documentation (fig. 49). Aucun atelier en activité durant le Haut-Empire n'est connu pour le moment entre le chef-lieu et les rives de l'étang de Thau. L'atelier le plus occidental est celui de Montbazin (Hérault). En Vaunage, en Lunellois et dans la région de Montpellier, les fouilles et les prospections, pourtant nombreuses, n'ont révélé que des témoignages sporadiques. On ne pourrait citer, avec quelques réserves du fait de l'arasement des vestiges, que l'ensemble comprenant une cuve entourée d'une quarantaine de dolia, qui a été dégagé à La Mougère de Vauguière, Mauguio (Hérault) (Roger, in: Massy dir., 1994, p. 109). L'étude du maillage de l'habitat rural révèle dans ces zones le rôle secondaire joué par les villae à côté des agglomérations secondaires. Cette partie de la cité aurait été moins attractive pour l'agriculture spéculative et le système de l'exploitation domaniale y aurait été moins développé (Favory et al., 1994, p. 206 et 209). La situation semble identique au nord de Nîmes, en Gardonnenque, si l'on se fie aux travaux de prospections encore largement inédits (Vidal et al., 1989).

La répartition des installations de production confirme donc la prééminence viticole de la frange rhodanienne de la cité, que laissait pressentir l'étude des ateliers d'amphores. Une telle situation pourrait être due 


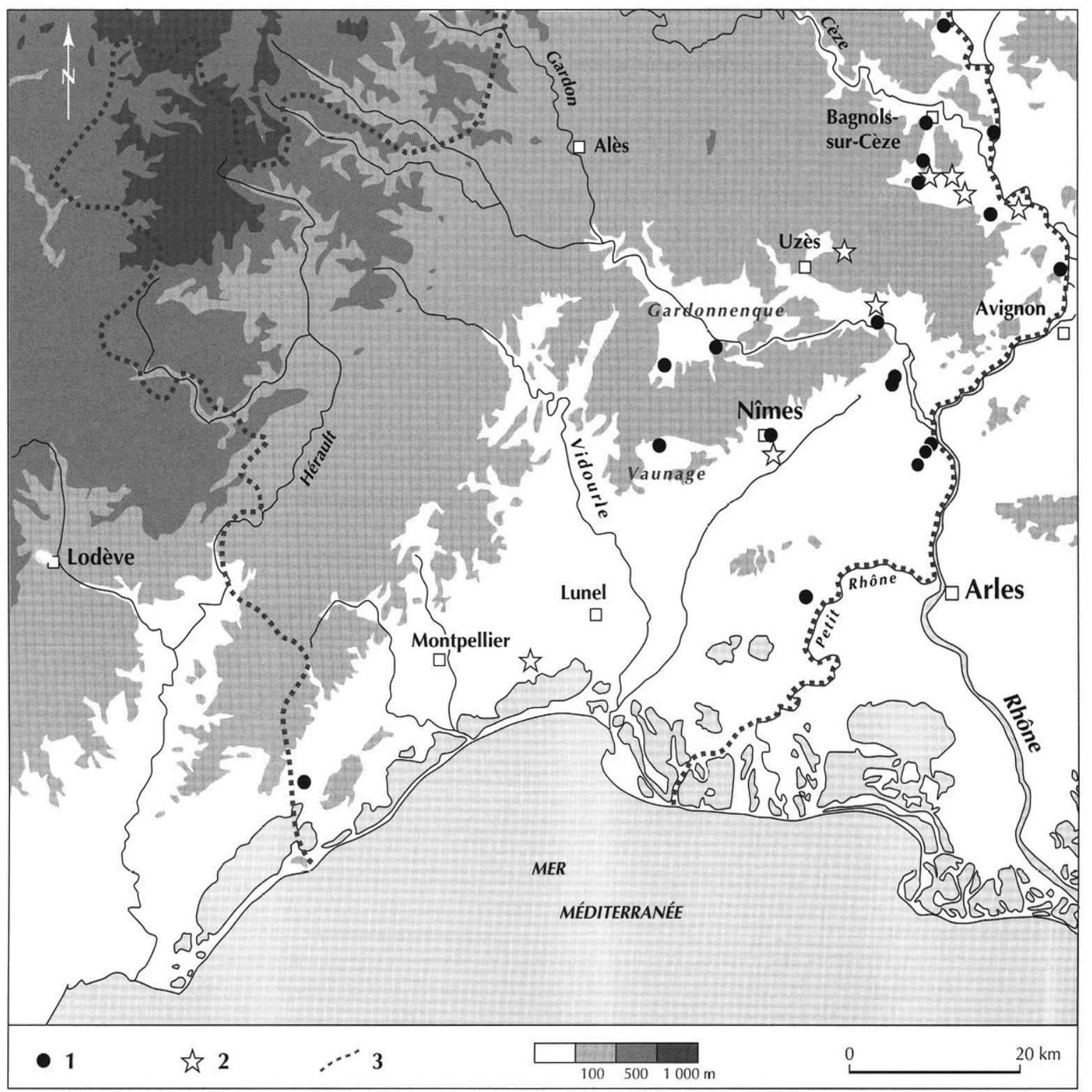

Fig. 49 - Installations vinicoles et ateliers d'amphores vinaires dans le territoire de la cilé de Nîmes : 1, ateliers d'amphores; 2, installations vinicoles; 3, limites supposées de la cité (dessin L. Buffat).

aux facilités pour écouler la production, par l'intermédiaire du fleuve ou de ses affluents qui permettaient de transporter selon l'opinion de Strabon « les plus forts tonnages" (Géographie, IV, 1, 14). Il est évidemment plus simple d'acheminer les amphores par voie d'eau, comme l'attestent les ateliers connus en bordure immédiate du Rhône ou du Gardon (Vieux-Cadenet à Chusclan et Font de Dringues à Vers-Pont-du-Gard). Cette situation avantageuse de la frange orientale de la cité joue d'autant plus que le Rhône ouvre les portes du marché du nord de la Gaule. À la lumière d'analyses physico-chimiques effectuées sur des amphores Gauloise 4, on pressent d'ailleurs l'importance de ce débouché pour les viticulteurs de l'ager nemausensis (Baudoux, 1996, p. 58-60).

On en terminera avec quelques documents échappant au domaine économique mais qui participent au dossier de la viticulture nîmoise. Un autel anépigraphe, décoré d'une grappe, a été retrouvé dans les thermes de l'oppidum de Gaujac, Gard (Charmasson, 1986, p. 21). À Nîmes, on connaît deux stèles funéraires, l'une dédiée à 
un certain Vallo (CIL XII, 4003), l'autre à un Secundus (Fiches, Veyrac, 1996, n 624,3 , p. 485), portant une serpette de vigneron.

\section{Dans la CITÉ de BÉZIERS}

Une distribution différentielle du vignoble est perceptible à l'échelle du territoire de la cité, comme le montre l'exemple du territoire nîmois. Des nuances de répartition doivent être envisagées aussi à l'intérieur même de microrégions. Dans la partie orientale du territoire nord-biterrois, en plein cœur de la zone productrice, $40 \%$ seulement des sites ruraux du Haut-Empire reconnus par prospection de surface livrent quelques indices d'une activité viticole: fragments de dolia abondants et très abondants, bâtiments de stockage individualisés (Mauné, 1998, p. 194-200).

Ces données ainsi que celles livrées par les fouilles les plus récentes permettent d'envisager un vignoble discontinu, polarisé autour de certains chefs-lieux d'exploitation, comme un caractère dominant du paysage rural. Il est encore difficile d'apprécier la surface occupée par les plantations mais, grâce à divers types d'approches, depuis les travaux de terrain jusqu'aux spéculations à partir des capacités de stockage, des chiffres commencent à être formulés. Les estimations, en tenant compte des rendements probables, varient entre quelques dizaines et la centaine d'hectares pour l'une ou l'autre des exploitations viticoles qui ont fait l'objet de tels calculs.

La fouille des installations de production permet d'entrevoir les éléments d'une hiérarchie entre les différentes exploitations viticoles. Ce sont les capacités des chais, à partir du nombre de dolia retrouvés, qui permettent de dégager, à titre provisoire, trois classes d'équipement ou trois niveaux de production : on pourrait ranger les plus modestes dans la catégorie de moins de 40 conteneurs (environ $600 \mathrm{hl} \pm 200$ ). L'exemple le plus clairement perceptible de ce type d'installation est celui de La Combe de Fignols à Péret (Hérault), déjà cité pour les interprétations discordantes qu'il suggère. Deux pressoirs (fig. 50), disposant d'une cuve de $85 \mathrm{hl}$, sont adaptés à une espérance de récolte de l'ordre de $320 \mathrm{hl}$ (20 dolia). Une catégorie moyenne est individualisée avec les ensembles de stockage d'une centaine de récipients. Un des exemples les plus significatifs est celui de la villa des Prés-Bas à Loupian (Hérault), dont le chai pouvait abriter environ $1500 \mathrm{hl}$. L'ultime catégorie est celle des

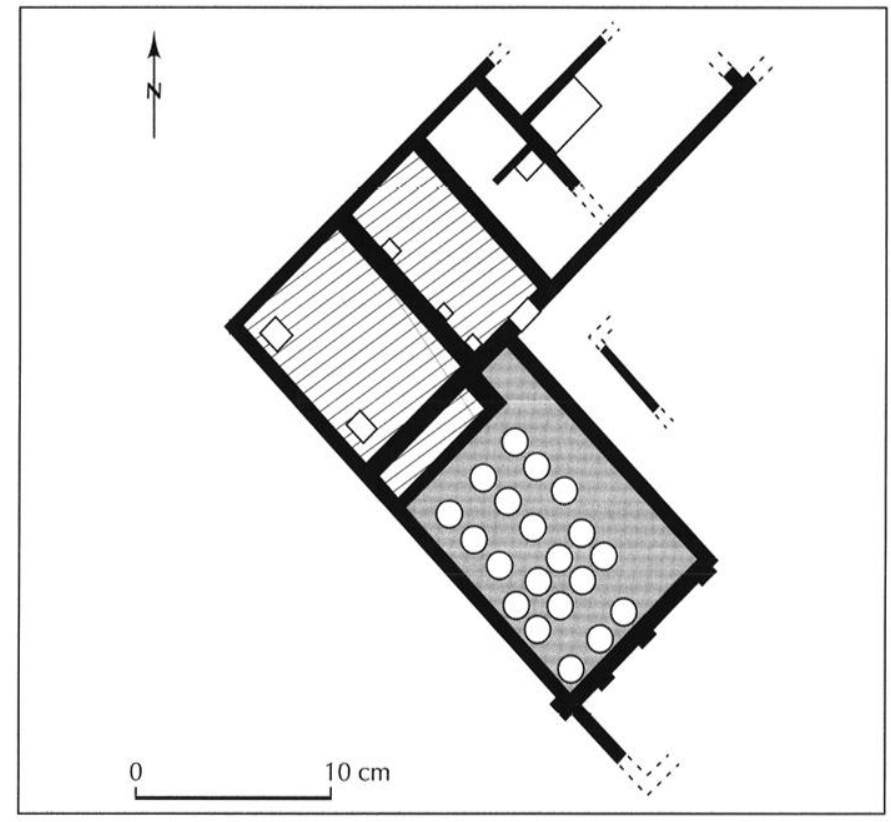

Fig. 50 - La ferme de La Combe de Fignols à Péret (Hérault), une huilerie ou une installation viticole? : en grisé, l'espace de stockage; en hachuré, les pressoirs (dessin P. Garczynski CEPAM-CNRS d'après C. Olive, complété).

très grandes exploitations viticoles dont les capacités dépassent ou doublent même le seuil de 100 dolia fixé pour les domaines d'ampleur moyenne. La Narbonnaise occidentale ne semblait pas connaître ce type d'installation attesté dans la vallée du Rhône ou en Provence orientale. Grâce à l'archéologie préventive, cette image s'est singulièrement transformée en quelques années avec la découverte en territoire biterrois de quelques-uns de ces ensembles destinés à une production en quantité, comme la villa de La Domergue à Sauvian, ou celle de Vareilles à Paulhan (Hérault).

$\mathrm{Au}$ moyen d'une meilleure connaissance des installations de production vinicole, de leur capacité et de leur répartition, mais aussi par l'estimation des surfaces plantées, on souhaiterait cerner les quantités produites à l'échelle de la cité dans le courant du Haut-Empire. L'étude des ateliers de fabrication d'amphores pourrait être une autre voie pour permettre d'étayer cette contribution notable de l'archéologie à l'histoire économique de la province. Cependant, des difficultés sont apparues lorsque l'on a tenté d'approcher le nombre des amphores produites dans des officines telles que Sallèlesd'Aude ou Loupian. Pour l'une comme pour l'autre, les récipients tournés annuellement - entre 3000 et 6000 amphores à Sallèles-d'Aude, soit une contenance de 
1000 à 2000 hl (Jamet, 2001) - ne permettent de fournir qu'un ou deux domaines de catégorie moyenne. Si l'observation vaut pour la vingtaine d'ateliers connus à ce jour dans toute la région languedocienne - soit une capacité totale de conditionnement de $60000 \mathrm{hl}$ au maximum -, l'offre ne peut rendre compte des besoins véritables de la viticulture régionale. S'agit-il là encore d'une lacune de l'inventaire archéologique ? Des travaux menés dans une microrégion comme celle de la vallée de l'Hérault pourraient le laisser penser, avec la découverte de nombreux ateliers inédits (Mauné, 2001a). On ne peut écarter cependant l'hypothèse qu'une part notable de la commercialisation du vin narbonnais ait pu se faire, dès le Haut-Empire, à l'aide de tonneaux.

La fin du $\mathrm{II}^{\mathrm{e}} \mathrm{s}$. et le $\mathrm{III}^{\mathrm{e}} \mathrm{s}$. constituent une période de rupture dans le développement de la viticulture née avec le Haut-Empire. L'abandon des installations de production apparaît comme un phénomène récurrent pour de très nombreux sites. Cependant, faute d'une véritable approche globale, il n'est pas toujours facile d'apprécier l'ampleur et la signification de cette " crise ". À cette époque, le maillage des établissements viticoles devient manifestement plus clairsemé. Cela est peut-être dû à unc tendance plus générale de mutation de la structure agraire (Raynaud, 1996, p. 204-209). Après la croissance paroxysmique $d u \mathrm{I}^{\text {cr }} \mathrm{s}$. de notre ère, les grands sites ruraux connaissent un essor régulier alors que les établissements les plus petits, ceux de moins de $5000 \mathrm{~m}^{2}$, voient leur nombre décroître. Dans la partie nordorientale du territoire biterrois, ces fermes ou petites villae qui n'atteignent pas le $\mathrm{IV}^{\mathrm{e}} \mathrm{s}$. constituent aussi la catégorie des sites ayant livré la plus forte proportion d'installations viticoles (Mauné, 1998, p. 194-200). À côté des effets de cette évolution structurelle perçue sur la très longue durée, on a cherché à dégager les signes de tendances plus conjoncturelles. L'idée d'une crise agricole spécifique a été avancée mais sans que l'on puisse conclure à une forte corrélation entre la vague d'abandon des sites ruraux et l'activité viticole (Mauné, 1998, p. 111-114).

\section{LA PERMANENCE DE LA PRODUCTION DURANT L'ANTIQUITÉ TARDIVE}

Comme le montrent les notices de sites, des découvertes en nombre croissant viennent étoffer le dossier de la viticulture tardive et du haut Moyen Âge. Pour asseoir une évidente continuité, on se contentait jusqu'à présent d'évoquer les vignes dans les descriptions des domaines de la Narbonnaise première faites par Sidoine Apollinaire (Épistula, II, 9, 1 et VIII, 4, 1), puis les mentions de sources plus tardives comme celles des chartes carolingiennes. Il est possible maintenant d'avoir recours à l'archéologie pour combler les lacunes de cette démonstration. Si aucun des ateliers fouillés en Narbonnaise n'est en activité après le $\mathrm{III}^{\mathrm{e}} \mathrm{s}$., certains sites de consommation livrent encore des Gauloise 4 pour le $\mathrm{IV}^{\mathrm{e}} \mathrm{s}$., voire le début du Ves. (Raynaud, 1990, p. 296 ; Piton, 1998, p. 113). Le débat est ouvert pour envisager une éventuelle poursuite de la fabrication ou admettre le caractère résiduel de ce matériel. Ce sont les fouilles des installations agricoles, étayées parfois par des études paléoenvironnementales, qui apportent les preuves évidentes d'une poursuite de la production viticole dans les villae et les fermes de la Narbonnaise première.

On ne peut nier que, comme dans la plupart des régions, les indices de production viticole tendent à se raréfier en Narbonnaise occidentale durant l'Antiquité tardive. Aucun cellicr avec des dolia defossa n'est attesté pour la période considérée. On ne connaît pas d'éléments de machinerie de pressoir. Seules quelques chapes solidement fondées équipent des pièces que l'on pense adaptées à l'étape du pressurage. Ces sont les cuves qui constituent les arguments les plus fréquents et les plus probants. D'un point de vue morphologique, elles n'ont guère évolué depuis le Haut-Empire. On trouve à la fois des cuves de petite et grande capacité, qui ne permettent pas de s'avancer à partir de ce seul élément sur une éventuelle réduction des quantités produites. Comme ailleurs, des conteneurs en bois, foudres et tonneaux, sont restitués pour la vinification, le stockage et la commercialisation. Il n'existe pas à ce jour de chai aux capacités élevées que l'on pourrait comparer aux plus grands ensembles des $\mathrm{I}^{\mathrm{er}}$ et $\mathrm{II}^{\mathrm{c}} \mathbf{s}$.

Même si les quantités produites ne peuvent être estimées, la documentation à notre disposition indique sans ambiguité qu'une large part $\mathrm{du}$ vin de la Narbonnaise occidentale est encore élaborée dans le cadre de l'exploitation domaniale. La plupart des installations connues ont été signalées dans des villae. On peut supposer que la construction de pressoirs et l'acquisition des récipients de stockage restent encore un lourd inves- 
tissement. À la différence des siècles passés cependant, la recherche d'un haut niveau de production n'est peutêtre plus la tendance majeure. Ceci expliquerait d'ailleurs la découverte de petites cuves isolées dans des sites de rang inférieur, fermes ou hameaux. Force est de constater que la viticulture n'occupe plus la place privilégiée qui était la sienne dans l'économie rurale du HautEmpire. 